\title{
RUN-OFF RISK AS A PART OF CLAIMS FLUCTUATION
}

\author{
By T. PentikäInen and J. Rantala \\ Ministry of Social Affairs and Health, Helsinki
}

\section{INTRODUCTION}

A conventional practice in standard risk theory considerations has been to assume that claims are paid immediately as they have incurred (see BPP, item 3.1c, BPP is used as an abbreviation for the book "Risk Theory", 1984 edition, by Beard, Pentikäinen, Pesonen). The delay of the claims settlement has been, of course, a central aspect in reserve calculation theory and practices, and numerous valuable works have been published on this topic in recent years. However, its regard in general model building and in risk theory considerations has gained little attention until recent years. The purpose of this paper is to contribute to this research work by discussing how the "run-off" risk, i.e., the variability due to the delay of the claims payment, could be incorporated into the standard risk theory models as a separate entry (see BPP, item 10.2e) and to find some evaluation of the order of magnitude of the "extra" (if any) fluctuation so rendered. We expect that the proposed technique can also be utilized in testing different reserve calculation methods and in comparing their effectiveness. The main ideas follow very much along the lines given by RANTALA in his doctoral thesis (1984).

One should appreciate the fact that any risk theory model can never be more than an idealization of real-life processes. An intricate problem for practitioners is to evaluate the uncertainties ensuing from the fact that the model, more or less, ignores or only approximates the factors affecting the real events, and that the practical applications are often based on and their necessary parameters estimated from observed data that are subject to random fluctuations and to many other kinds of uncertainties. The problem complex of the run-off risk, when understood in a broad sense, is so wide that it requires a series of studies, and this paper should be regarded as a first step only. The posing of the problem follows the conventional risk theory approach by using the mixed compound Poisson process further allowing for long-term variations of risk exposure ("cycles"), and now extending the model to cope with the delayed settlement of the claims. At this stage of the on-going researchwork the impact of the parameter estimation is excluded from consideration. Therefore, our results and the numerical examples, as given in what follows, do not describe the total uncertainty of the claims or the reserves.

The structure of the paper is the following. First, the main assumptions are given in Chapter 1, then the effect of the run-off phenomenon on the variance of the claims fluctuation is evaluated for the going-concern case in Chapter 2 and for the break-up case in Chapter 3. Some numerical examples will be given in Chapter 4 and finally in Chapter 5 the same effect is considered by using the simulation technique, which allows for more general assumptions on inflation 
and the other pertinent features involved. As mentioned above, the handling of the parameter estimation problem is deferred to a future paper. The estimation of claims reserves has already been explored in numerous meritorious treatices, see e.g., the Survey published by the Nationale Nederlande (1981), the GISG Working Party summary paper of the British actuaries (1983), TAYLOR (1986) and many others. One of the early papers on the building of "a run-off theory" was published by PHiLipson (1965). Norberg (1985) when dealing with estimation techniques makes use of partially similar development functions as will be employed in this paper.

\section{DEFINITIONS, NOTATIONS}

\subsection{Paid and Not Paid Claims}

We are going to consider a claims process $\boldsymbol{X}$. (The stochasticity of the variables is indicated by boldface letters.) The issue is the fact that it always takes some time until a claim is notified to the insurer after it has been incurred. A further time delay occurs until it is paid. For the claims which are not notified a statistically constructed reserve should be established, the so called IBNR-reserve (incurred but not reported). Some insurers also use statistical, collective reserves for some classes of outstanding claims, irrespective of whether or not they are notified, in order to rationalize the administration (and possibly for stabilizing the flow of business). Furthermore, often the information concerning already notified but still outstanding claims may be defective, which precludes the insurer from making an exact case estimate. So is it e.g., if the claim depends on an on-going court trial. All these reasons occasion uncertainty in the evaluation of the business outcomes. An inaccuracy in the claims reserve reflects as an inaccuracy in the profit so long as the claim is not finally settled. The purpose of this paper is to evaluate this uncertainty.

In the following we assume that the claims are either paid or outstanding. For brevity we (somewhat loosely) define as "paid" a claim which is notified and is so well documented that its final amount can be reliably determined. All other claims are "not paid", i.e., either IBNR, collectively reserved or defectively informed. Of course, it depends on the practice and on the purposes of the considerations just how the interdisciplinary boundary between "paid" and "not-paid" claims should be defined. For example, there is nothing which prevents the treating of the IBNR-claims only and ignoring the other uncertainties which are beyond the scope of this consideration. Another alternative is to separate the (unknown) exact value of the already notified claims and its estimate and to handle the difference as if it were an unknown claim. We also use the terminology as if only one payment would correspond to each claim. Another interpretation would be to take "claim" as "claim payment". Then e.g., the number of paid claims should be read as the number of claim payments. Of course, the distributions and some of the initial data depend on the definitions which are selected in any particular application, but the theoretical apparatus is very much the same, even though the handling of data, parameter estimation, etc. differ. 


\subsection{Cohort}

The claims are grouped as "cohorts" according to the year of origin $t_{0}$, during which they have been incurred. The payment of the claims of the cohort are distributed to years $t_{0}, t_{0}+1, \ldots, t_{0}+u, \ldots, t_{0}+T$, as shown in fig. $1.1 T$ is the maximum time for the claims settlement. The "current time" (or the year of observation) is denoted by $t$ and the elapsed time from the birth of the cohort by $s=t-t_{0}$. The claims of the cohort $t-s$ paid in year $t-s+u$ are denoted by $\boldsymbol{X}(t-s, u, u)$ and those paid together in years $t-s+u_{1}, ., t-s+u_{2}$ by

$$
\boldsymbol{X}\left(t-s, u_{1}, u_{2}\right)=\sum_{u=u_{1}}^{u_{2}} \boldsymbol{X}(t-s, u, u)
$$

Notations. As above for $X$, an argument $\left(t-s ; u_{1}, u_{2}\right)$ will be used for the variables to indicate that the event to which the variable is related has occured in year $t-s$ and its settlement happens in the period ranging from the beginning of year $t-s+u_{1}$ until the end of year $t-s+u_{2}$, e.g., $n\left(t-s ; u_{1}, u_{2}\right)$ for the number of clarms. Furthermore, the argument $(t-s)$ indicates the whole cohort, e.g., $X(t-s)$ or $n(t-s)$ represent the total amount or the total number of claims respectively of the cohort of the year $t-s$.

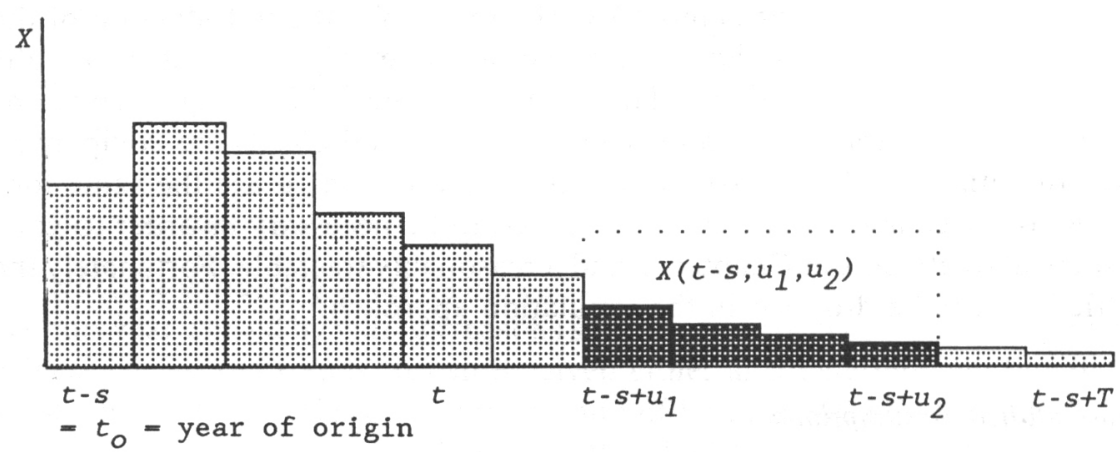

FIGURE 11 A cohort of claims The current year is denoted by $t$ and the year of the origin of the cohort by $t-s$ The pillars describe the distribution of the settlement of the claims inside the cohort

\subsection{Basıc Assumptions}

We shall choose our assumptions so that $\boldsymbol{X}(t ; 0, T)$ will be a mixed compound Poisson process (in a broad sense).

Firstly, the intensity of the occurrences of claims is allowed to change from year to year, hence from cohort to cohort. This effect is incorporated into the model via a randomly varying Poisson parameter $\boldsymbol{n}(t-s)$ (expected number of claims, BPP, item $2.7 \mathrm{c}$ )

$$
n(t-s)=n \cdot q(t-s) \cdot I_{n}(t-s)
$$


where $n$ is a non-random volume parameter and the stochasticity is introduced via the structure variable $q(t-s)$. The parameter $n$ indicates, in fact, the expected number of claims for the whole cohort of a suitably chosen basis year $t_{b}$.

$q(t-s)$ is "a structure variable" indicating variations in the risk exposure from cohort to cohort, as shall be specified in item 1.5; (see BPP, item 2.7c). $I_{n}(t-s)$ is a volume index indicating the volume of the risk portfolio for the cohort of year $t-s$ compared with the basic year $t_{b}$, hence scaled to have $I_{n}\left(t_{b}\right)=1$. More details are discussed in item 1.6. This index allows, if so desired, for a regard for the growth of the business volume, e.g., occasioned by the acquisition of new policies.

It is often useful to choose the current year $t$ as the basic year of indexes. Then the monetary quantities are obtained in the current value of money.

Secondly, the amounts of individual claims are assumed to change from year to year so that the shape of the d.f. $S$ of the claim size $Z$ is preserved but the mean claim size $E \boldsymbol{Z}$ varies according to rules which will be specified later.

Furthermore, the distribution of the claims payment delays inside each cohort is controlled by functions $g_{n}(u), g_{m}(u)$ and $g_{x}(u)$, which may be separately given for the claims numbers $(n)$, average claim sizes $(m)$ and for the total amount of claims $(X)$ paid in the $u$ th development year. These functions will also be specified later in more detail. We assume that these functions are common for all cohorts, i.e., independent of the time points $t-s$. The reason for the introduction of three development functions instead of a conventional one only $\left(g_{x}(u)\right)$ is to also allow for the variation of the average claim size inside the cohorts, e.g., trends up or down which may appear when delayed and early paid claims are compared.

On the other hand, the assumption that the functions $g$ are the same for all cohorts is, of course, a restriction. It proved to be necessary in order to get the theoretical treatments in Chapters 2 and 3 reasonably tractable. Fortunately these restrictions can be dropped in the simulation approach.

\subsection{Detailed Assumptions}

We shall now present our assumptions in a more detailed form as follows:

The variables $\boldsymbol{X}(t-s ; u, u)$ for $u=0,1, \ldots, T$ are conditionally, for a given $q(t-s)$, mutually independent and distributed according to the compound Poisson law (BPP, item 3.2b) having the parameter ( $=$ the expected number of claims) (cf. (1.2))

$$
\begin{aligned}
\boldsymbol{n}(t-s ; u, u) & =\boldsymbol{n}(t-s) \cdot g_{n}(u) \\
& =n \cdot I_{n}(t-s) \cdot \boldsymbol{q}(t-s) \cdot g_{n}(u) .
\end{aligned}
$$

$g_{n}(u)$ is "a development function" of the claims numbers inside the cohort:

$$
g_{n}(u)=E n(t-s ; u, u) / E n(t-s ; 0, T) .
$$

Let $S(Z ; t-s ; u, u)$ be the d.f. of the sizes of the claims that occurred in year $t-s$ and were paid in year $t-s+u$. We assume that the shape of this distribution 
is the same for all values of $t-s$ and $u$, but the scale is changed so that the expected value of the claim size variable $Z$ is:

$$
E Z(t-s ; u, u)=m \cdot I_{m}(t-s) \cdot g_{m}(u)
$$

where, in addition to those given above, the following concepts and notations are used:

$m$ is the mean size of the claims of the cohort of the basic year $t_{b}$ and paid in the same year.

$I_{m}(t-s)$ is an index for the mean size of the claims of the cohort $t-s$ and paid in the same year $t-s$ (indicating the effect of inflation and other potential movements, again $\left.I_{m}\left(t_{b}\right)=1\right)$.

$g_{m}(u)$ represents the development of the average claim size inside a cohort. It indicates (1) the effect of inflation compared with its level at the first year of the cohort $(=t-s)$, and (2) changes (if any) for any other reason which may result in upward or downward trends in the real size of delayed claims. It will be scaled to have $g_{m}(0)=1$.

Note that the payments $\boldsymbol{X}(t-s ; u, u)$ for consecutive development years $u$ are correlated owing to the assumption that the structure variable $q$ is the same for the whole cohort, but they are conditionally uncorrelated when $q$ is given. In practice there may appear circumstances that also render other correlations, for instance, if the working capacity of the claims settlement is changed in some particular year, it likely renders a negative correlation. These kinds of features are not accounted for in our paper, but supposedly they and other similar changing conditions can be properly observed on a case basis, if deemed necessary.

\subsection{The Structure Variable $q$}

The structure variable $q$ controls the variations of risk exposure from cohort year to cohort year caused by various reasons such as the impact of weather conditions, business cycles and trends. It can be given either as a deterministic function or as a stochastic time series or as any mixture of both. It will be exemplified as an autoregressive time series in Chapter 4 . We shall limit our considerations only to the cases where $q(t)$ is weakly stationary, i.e., the following characteristics exist and are finite:

$$
E q=1 ; \quad \operatorname{Var} q=\sigma_{q}^{2} ; \quad \operatorname{Cov}(\boldsymbol{q}(t), \boldsymbol{q}(t+i))=\gamma(i)
$$

\subsection{Inflation}

Inflation between the cohorts is controlled by the above index $I_{m}$. We assume that the inflation between the development years inside the cohort is amalgamated into the development function $g_{m}(u)$.

Note that we need not assume that the inflation between the cohorts and inside the cohorts should be the same. Policy conditions and other reasons may cause differences, for which our model allows. A restriction is, however, that the inflation rates within the cohorts, as amalgamated in the development functions $g_{m}$, are 
assumed to be the same for all cohorts as was stated in item 1.3. This assumption can be, however, relaxed in the simulation as presented in Chapter 5.

\subsection{Function $g_{x}(u)$}

We further introduce a third development function to indicate the development of the total amount of claims inside any cohort. It can be defined by means of the other two as follows

$$
g_{x}(u)=g_{n}(u) g_{m}(u)
$$

Thus $E \boldsymbol{X}(t-s ; u, u)=n m I_{n}(t-s) I_{m}(t-s) g_{x}(u)$. When any two of the development functions are given, then the third is determined from (1.7).

REMARK 1. Often the distribution of the total payments inside the cohort is readily available and therefore used as a basic development function. It is in terms of our concepts

$$
E X(t-s ; u, u) / E X(t-s ; 0, T)=g_{x}(u) / G_{x}(T)
$$

where $G_{x}$ is the accumulated development function obtained from $g_{x}(u)$ by summation (see (1.12) below).

REMARK 2. Possibly only the function $g_{x}(u)$ may be available, not the claim size development $g_{m}(u)$. This can be the case e.g., in ingoing reinsurance. Then e.g., taking $g_{m}(u) \equiv 1$ avoids the model breaking down. It implies $g_{n}(u)=g_{x}(u)$. Another alternative is to assume that the inflation within the cohorts and between them is the same, which makes it possible to construct $g_{m}(u)$ accordingly. Of course, some of the model outcomes are sensitive to these kinds of simplifications, but likely not in a fatal way, as some examples given later seem to indicate.

\subsection{The Moments of the Claim Size Distribution}

The moments about zero of the claim size distribution are, according to the assumptions (item 1.4) that the scaling only is changed from year to year, as follows:

$$
\begin{aligned}
a_{1}(t-s ; u, u) & =\int_{0}^{\infty} Z^{\prime} d S(Z ; t-s ; u, u) \\
& =a_{\imath} \cdot I_{m}(t-s)^{\prime} \cdot g_{m}(u)^{t} \quad(i=1,2,3, \ldots)
\end{aligned}
$$

where $S$ is the distribution function of the claim size and $a_{i}=a_{i}\left(t_{b} ; 0,0\right)$ is a basic amount calculated from (1.9) replacing there $S$ by $S\left(Z ; t_{b} ; 0,0\right)$ for the basic year $t_{b}$ of the indexes. 


\subsection{Composition of the Development Years}

Our assumptions imply that the sum variable (1.1) is again a mixed compound Poisson variable having the parameter

$$
n\left(t-s ; u_{1}, u_{2}\right)=\sum_{u=u_{1}}^{u_{2}} n(t-s ; u, u)
$$

Here and in the sequel the $n$ 's not printed by bold-face letters denote the expected values $n()=E n()$ of the various claim numbers.

REMARK. If $g_{m}(u)$ were $\equiv 1$, then the claim size distributions of the development years $u$ of each cohort separately would be identical. Then we could reverse the order of the assumptions, i.e., if the cohort variable $\boldsymbol{X}(t-s ; 0, T)$ is assumed to be compound Poisson distributed for any fixed $q$ and the development times are i.i.d. random variables then the development variables $\boldsymbol{X}\left(t-s ; u_{1}, u_{2}\right)$ for a given $q(t-s)$ are i.i.d. compound Poisson variables (see KARLSSON 1974).

The moments related to any development period $\left(u_{1}, u_{2}\right)$ are obtained from the equation (see BPP, item 3.7a)

$$
n\left(t-s ; u_{1}, u_{2}\right) \cdot a_{i}\left(t-s ; u_{1}, u_{2}\right)=\sum_{u=u_{1}}^{u_{2}} n(t-s ; u, u) \cdot a_{i}(t-s ; u, u)
$$

which expresses the characteristics of a sum variable in terms of the corresponding characteristics of the component variables.

\subsection{Accumulated Development Functions}

Accumulated development functions are denoted by corresponding capital letters and defined as follows

$$
G_{*}(u)=\sum_{v=0}^{u} g_{*}(v) \quad(\text { replace } * \text { here by } n, x \text { respectively }) .
$$

These functions are scaled by the conditions (cf. (1.4))

$$
G_{n}(T)=1 ; \quad g_{m}(0)=1 .
$$

Owing to (1.7) $g_{x}(u)$ is then uniquely determined. In addition, we define $G_{*}(u)=0$ for $u \leqslant 0$.

\subsection{The Mean Total Claim Amount}

The mean total amount of claims for the cohort interval $u_{1}, u_{2}$ is now

$$
E X\left(t-s ; u_{1}, u_{2}\right)=X_{0} \cdot I_{x}(t-s) \cdot\left[G_{x}\left(u_{2}\right)-G_{x}\left(u_{1}-1\right)\right]
$$

where

$$
X_{0}=n m \quad \text { and } \quad I_{x}=I_{n} I_{m}
$$


Here $X_{0}$ is the average basic amount of the total amount of claims for the cohort $t_{b}$, (see BPP, item 3.3c). $I_{x}$ transforms the business volume and the monetary values to the level of the birth of the cohort $t-s$, and the difference of the $G_{x}$ functions in (1.14) allocates the shares of the claims to the period $u_{1}, u_{2}$ adjusting also the monetary value to correspond to the level of the time payment.

\subsection{The Variances of the $X$ Variables}

The variances of the $\boldsymbol{X}$ variables are still needed as primary building blocks for the further considerations. We have, according to the standard risk theory (see BPP, item 3.3c) and by using the above concepts and notations, the variance for the one year payment

$$
\begin{aligned}
\operatorname{Var} X(t-s ; u, u)= & n(t-s ; u, u) \cdot a_{2}(t-s ; u, u)+\sigma_{q}^{2} \cdot(E X(t-s ; u, u))^{2} \\
= & n \cdot a_{2} \cdot I_{n}(t-s) \cdot g_{n}(u) \cdot I_{m}(t-s)^{2} \cdot g_{m}(u)^{2} \\
& +\sigma_{q}^{2} \cdot n^{2} \cdot m^{2} \cdot I_{x}(t-s)^{2} \cdot g_{x}(u)^{2} .
\end{aligned}
$$

Introducing the notations

$$
v_{0}=n \cdot a_{2} ; \quad v_{q}=\sigma_{q}^{2} \cdot X_{0}^{2}
$$

for the basic amounts of the "pure Poisson" variation and of the structure variation, we can write the above expression in the form

$$
\begin{aligned}
\operatorname{Var} \boldsymbol{X}(t-s ; u, u)= & v_{0} \cdot I_{x}(t-s) \cdot I_{m}(t-s) \cdot g_{m}(u) \cdot g_{x}(u) \\
& +v_{q} \cdot I_{x}(t-s)^{2} \cdot g_{x}(u)^{2}
\end{aligned}
$$

Let

$$
G_{m x}(u)=\sum_{v=0}^{u} g_{m}(v) \cdot g_{x}(v)
$$

be another development function. Then the corresponding variance for the payment period $u_{1}, u_{2}$ can be written as

$$
\begin{aligned}
\operatorname{Var} \boldsymbol{X}\left(t-s ; u_{1}, u_{2}\right)= & v_{0} \cdot I_{x}(t-s) \cdot I_{m}(t-s) \cdot\left[G_{m x}\left(u_{2}\right)-G_{m x}\left(u_{1}-1\right)\right] \\
& +v_{q} \cdot I_{x}(t-s)^{2} \cdot\left[G_{x}\left(u_{2}\right)-G_{x}\left(u_{1}-1\right)\right]^{2}
\end{aligned}
$$

in a similar way as (1.17) by using the rule (1.11) (see BPP, item 3.7).

\subsection{The Yield of Interest}

The yield of interest is provided to be given as a separate entry into the comprehensive models and it is not needed to be taken into account in the context of the claims variable (see BPP, item 10.2a). However, for calculation of the necessary premium rates and for evaluation of the proper amount of the reserve for outstanding claims an assumption of the rate of interest is needed, if the discounting of future yield of interest is considered appropriate. We use a constant rate of interest $i_{i}$ in Chapters $2-4$ and a randomly varying rate for simulations in Chapter 5 . The discounting factor is denoted by

$$
v=1 /\left(1+i_{i}\right) \text {. }
$$




\subsection{The Discounted Future Claims Expenditure}

The model is now extended to allow for the discounting of the future payments. We shall need only summations from the end of the current year $t$ to the end of each cohort:

$$
\begin{aligned}
E \boldsymbol{X}_{v}(t-s ; s+1, T) & =\sum_{u=s+1}^{T} v^{\left(u-s-\frac{1}{2}\right)} E \boldsymbol{X}(t-s ; u, u) \\
& =\sum_{u=s+1}^{T} X_{0} v^{\left(u-s-\frac{1}{2}\right)} I_{x}(t-s) g_{x}(u) \\
& =X_{0} I_{x}(t-s) \bar{G}_{x}(s)
\end{aligned}
$$

where in an analogy with (1.12)

$$
\bar{G}_{x}(s)=\sum_{u=s+1}^{T} v^{\left(u-s-\frac{1}{2}\right)} g_{x}(u)
$$

is a modified accumulated development function. The subscript $v$ of the $X$ indicates the discounting and the bar over $G$ both the discounting and the fact that this function is a complement of the function $G_{x}(s)$ as given by (1.12). However, their sum $\bar{G}+G$ is not usually equal to 1 , unless $v=1$ and $g_{m}(u)$ is equal to 1 for all the $u$ values.

Note that the discounting is made to relate to current yeat $t$.

\subsection{The Reserve of Outstanding Claims}

The reserve of outstanding claims has a central role in all run-off issues. The run-off errors understandingly depend on the rules and practices applied in reserve calculations. As we already stated above, the estimation problems of the parameters introduced above will not be dealt with. Instead we shall assume that the claims reserve in the year $t$ for the "unpaid" claims for the cohort $t-s$ (as defined in item 1.1 above) is given by a general formula (see RANTALA 1984 , p. 38)

$$
C(t, t-s)=c_{p}(s) \cdot P(t-s)+c_{x}(s) \cdot \boldsymbol{X}(t-s ; 0, s), \quad s=0,1,2, \ldots, T-1
$$

where $P(t-s)$ is the premium related to the cohort $t-s$ and the coefficients $c_{p}$ and $c_{x}$ can be interpreted as weights to balance the general past experience (the first term) and the fresh experience obtained just from this particular cohort up to year $t$ (the second term) in the evaluation of the reserve. In order not to have any safety margin $(+$ or - ) hidden in the claims reserve we demand that the expected value of $C(t, t-s)$ should be equal to the expected value of outstanding unpaid claims:

$$
E C(t, t-s)=E X_{v}(t-s ; s+1, T), \quad s=0,1,2, \ldots, T-1 .
$$

Otherwise these coefficients are freely eligible for applications.

Many of the generally used rules are covered by the formula (1.23) as special cases (see Survey of the Nationale Nederlande 1981). If $c_{x} \equiv 0$, then we have 
a "premium based" rule, which is often used as a first approximation. The choice $c_{p} \equiv 0$ represents a method which is close to the various chain ladder approaches and the combinations of the two terms represents a variety of mixtures of these alternatives among them rules of experience rating character as will be shown in Chapter 4.

REMARK. The above formula (1.23) was adopted mainly to find a concrete illustration, not claiming that it would be the most suitable in all environments. Other reserve calculation procedures can likely be treated by using the same technique with obvious modifications. Furthermore, if there had occurred significant changes or disturbances in the claim process (renewed policy conditions, changed judicial practice, etc.) or in the claims settlement handling, they should be properly observed by making the necessary corrections to the relevant formulae.

The total reserve consists of the current cohort reserves

$$
C(t)=\sum_{s=0}^{T-1} C(t, t-s)
$$

The premium income will be defined for our applications by means of the formula

$$
\begin{aligned}
P(t-s) & =\sum_{u=0}^{T} v^{\left(u+\frac{1}{2}\right)} E X(t-s ; u, u) \\
& =I_{x}(t-s) P_{0}
\end{aligned}
$$

where

$$
P_{0}=X_{0} \cdot \bar{G}_{x}(-1) \text {. }
$$

Note: An alternative approach to the conditions (1.24) would be to provide only the unbiasedness for the total amount of the claims reserve:

$$
E C(t)=\sum_{s=0}^{T-1} E \boldsymbol{X}_{v}(t-s ; s+1, T)
$$

Then the cohort reserves may be biased. This is the case in the reserving rule "opt 1" which will be introduced for numerical examples in Chapter 4.

\section{RUN-OFF ERROR, GOING-CONCERN}

\subsection{The Problem}

We are now ready to examine the effects of a run-off pattern on the claims expenditure in conventional income statements. The claims expenditure is given as the amount of claims paid in the accounting year $t$ irrespective of the year of the origin of the claims added by the increment of the reserve of outstanding claims:

$$
\boldsymbol{X}_{p}(t)=\sum_{s=0}^{T} \boldsymbol{X}(t-s ; s, s)+C(t)-C(t-1)
$$


The run-off errors consist of the differences between the eventually settled amounts of the claims and their estimates such as are taken into the reserve of the outstanding claims. They are not revealed until in the year of the final settlement. Any amendment in the reserve calculation affects the profit or loss for the year during which it is made.

The errors and effects of concern will be measured by means of the variances of the relevant variables. Therefore, we shall first derive a programmable expression for the variance of $\boldsymbol{X}_{p}$.

Note: It would be possible to tender the following presentation in a more compact form by using overall vector notations for the variable sets as will be demonstrated in the Appendix 1. We chose, however, a more conventional approach which may be easier to read because the background tie-ins are more clearly recognizable.

\subsection{The Variance of the Claims Expenditure}

The expression (2.1) can be written by using the definitions (1.23) and (1.25) as follows

$$
\begin{aligned}
\boldsymbol{X}_{p}(t)= & \sum_{s=0}^{T} \boldsymbol{X}(t-s ; s, s) \\
& +\sum_{s=0}^{T-1}\left(c_{p}(s) \cdot P(t-s)+c_{x}(s) \cdot \boldsymbol{X}(t-s ; 0, s)\right) \\
& -\sum_{s=1}^{T}\left(c_{p}(s-1) \cdot P(t-s)+c_{x}(s-1) \cdot \boldsymbol{X}(t-s ; 0, s-1)\right) .
\end{aligned}
$$

In order to get the lengths of the summations and later the corresponding vectors to have a uniform dimension $T+1$ we introduce the conventions

$$
\begin{aligned}
c_{p}(u)=c_{x}(u)=0 & \text { for } u<0 \text { and for } u>T-1, \\
G_{x}(u)=G_{m x}(u)=0 & \text { for } u<0, \\
X(\cdot, \cdot, u)=0 & \text { for } u<0 .
\end{aligned}
$$

Since $\boldsymbol{X}(t-s ; 0, s)=\boldsymbol{X}(t-s ; 0, s-1)+\boldsymbol{X}(t-s ; s, s)$, the above expressions can be reordered:

$$
\begin{aligned}
\boldsymbol{X}_{p}(t)= & \sum a(s) \cdot \boldsymbol{X}(t-s ; s, s)+\sum b(s) \cdot \boldsymbol{X}(t-s ; 0, s-1) \\
& +\sum k(s) \cdot P(t-s) \\
\equiv & \boldsymbol{S}_{1}+\boldsymbol{S}_{2}+S_{3},
\end{aligned}
$$

where

$$
\begin{gathered}
a(s)=1+c_{x}(s) ; \quad b(s)=c_{x}(s)-c_{x}(s-1) ; \\
k(s)=c_{p}(s)-c_{p}(s-1) .
\end{gathered}
$$


All the summations are from 0 to $T$ here and in the sequel unless otherwise stated. The $S$ 's stand briefly for the three sums above. In terms of them we have

$$
\operatorname{Var} \boldsymbol{X}_{p}=\operatorname{Var} \boldsymbol{S}_{1}+\operatorname{Var} \boldsymbol{S}_{2}+2 \operatorname{Cov}\left(\boldsymbol{S}_{1}, \boldsymbol{S}_{2}\right) \text {. }
$$

The terms will now be calculated each in turn. Firstly

$$
\begin{aligned}
\operatorname{Var} \boldsymbol{S}_{1}= & \sum a(s)^{2} \cdot \operatorname{Var} \boldsymbol{X}(t-s ; s, s) \\
& +\sum_{u \neq s} a(s) \cdot \boldsymbol{a}(u) \cdot \operatorname{Cov}(\boldsymbol{X}(t-s ; s, s), \boldsymbol{X}(t-u ; u, u)) .
\end{aligned}
$$

The variance terms are readily available from (1.19). In addition we need, here and in the sequel, covariances, which will be derived next.

\subsection{The Covariances}

The covariances which are building blocks in our considerations are of the form

$$
\operatorname{Cov}\left(\boldsymbol{X}\left(t-s ; u_{1}, u_{2}\right), \boldsymbol{X}\left(t-w ; v_{1}, v_{2}\right)\right) .
$$

For $s \neq w$ we have (see RANTALA (1984) and equations $(2.3,3.4)$ ) and (1.14) above

$$
\begin{aligned}
\operatorname{Cov}(\cdot, \cdot)= & \gamma(|s-w|) E \boldsymbol{X}\left(t-s ; u_{1}, u_{2}\right) E \boldsymbol{X}\left(t-w ; v_{1}, v_{2}\right) \\
= & \gamma_{0}(|s-w|) \cdot\left[I_{x}(t-s) \cdot\left(G_{x}\left(u_{2}\right)-G_{x}\left(u_{1}-1\right)\right)\right] \\
& \cdot\left[I_{x}(t-w) \cdot\left(G_{x}\left(v_{2}\right)-G_{x}\left(v_{1}-1\right)\right)\right]
\end{aligned}
$$

where in terms of the covariances $\gamma$ of the structure variable (see (1.6))

$$
\gamma_{0}(|s-w|)=\gamma(|s-w|) X_{0}^{2} .
$$

Providing $u_{1} \leqslant u_{2}<v_{1} \leqslant v_{2}$ the covariance expression (2.9) also holds for $s=w$.

\subsection{The variance Var $S_{1}$}

The variance $\operatorname{Var} S_{1}$ is obtained by substituting (1.17), (2.9) and (2.10) into (2.7)

$$
\begin{aligned}
\operatorname{Var} S_{1}= & \sum a(s)^{2} v_{0} \cdot I_{x}(t-s) \cdot I_{m}(t-s) \cdot g_{m}(s) \cdot g_{x}(s) \\
& +\sum a(s)^{2} \cdot v_{q} \cdot I_{x}(t-s)^{2} \cdot g_{x}(s)^{2} \\
& +\sum \gamma_{0}(|s-u|)\left[a(s) \cdot I_{x}(t-s) \cdot g_{x}(s)\right] \\
& \cdot\left[a(u) \cdot I_{x}(t-u) \cdot g_{x}(u)\right]
\end{aligned}
$$

\subsection{Vector Representation}

The formulae tend to become long and complicated even though they are simple in concept. Therefore, we shall adopt vector notations, which help to maintain a survey over the structures and which are also useful in computer programming.

The first two terms of (2.11) can be written in the form

$$
v_{0} \cdot V_{a x}^{\prime} V_{a m}+v_{q} \cdot V_{a x}^{\prime} V_{a x}
$$


where the capital letter symbols represent $T+1$ dimensional column vectors:

$$
\begin{aligned}
V_{a x} & =\left(V_{a x}(s)\right)=\left(a(s) I_{x}(t-s) g_{x}(s)\right)^{\prime} \\
V_{a m} & =\left(V_{a m}(s)\right)=\left(a(s) I_{m}(t-s) g_{m}(s)\right)^{\prime}
\end{aligned}
$$

and $V_{1}^{\prime} V_{2}$ are the conventional (scalar) vector products $=\sum s V_{1}(s) V_{2}(s)$.

Note that the vectors depend also on the current time $t$.

It is rewarding to introduce a short operative symbol for the covariance summation:

$$
\operatorname{CS}\left[V_{1}, V_{2}\right]=\sum_{i=1}^{T} \gamma_{0}(i) \sum_{\substack{|s-u|=i \\ 0 \leq s, u \leq T}} V_{1}(s) \cdot V_{2}(u)
$$

where $V_{1}$ and $V_{2}$ are two vectors of length $T+1$ and the elements are indexed from 0 to $T$.

Hence we can write

$$
\operatorname{Var} S_{1}=v_{0} V_{a x}^{\prime} V_{a m}+v_{q} V_{a x}^{\prime} V_{a x}+\operatorname{CS}\left[V_{a x}, V_{a x}\right]
$$

(Cf. equation (3) of Appendix 1.)

\subsection{Summary}

The other terms in (2.6) can be handled in a similar way and the following formula results:

$$
\begin{aligned}
\operatorname{Var} \boldsymbol{X}_{p}(t)= & v_{0}\left(V_{a x}^{\prime} V_{a m}+W_{b m}^{\prime} W_{b I}\right)+v_{q}\left(V_{a x}^{\prime} V_{a x}+W_{b x}^{\prime} W_{b x}+2 V_{a x}^{\prime} W_{b x}\right) \\
& +\operatorname{CS}\left[V_{a x}, V_{a x}\right]+\operatorname{CS}\left[W_{b x}, W_{b x}\right]+2 \operatorname{CS}\left[V_{a x}, W_{b x}\right]
\end{aligned}
$$

where the vectors involved are

$$
\begin{aligned}
V_{a x} & =\left(a(s) \cdot I_{x}(t-s) \cdot g_{x}(s)\right)^{\prime} \\
V_{a m} & =\left(a(s) \cdot I_{m}(t-s) \cdot g_{m}(s)\right)^{\prime} \\
W_{b x} & =\left(b(s) \cdot I_{x}(t-s) \cdot G_{x}(s-1)\right)^{\prime} \\
W_{b m} & =\left(b(s) \cdot I_{m}(t-s) \cdot G_{m x}(s-1)\right)^{\prime} \\
W_{b I} & =\left(b(s) \cdot I_{x}(t-s)\right)^{\prime} .
\end{aligned}
$$

For convenience of the reader we shall recap by stating that the functions $a(s)$ and $b(s)$ are given by (2.5), the indexes $I$ in items 1.3 and $1.4, g_{x}$ and $g_{m}$ in (1.7) and in item 1.4, $G_{m x}$ in (1.18) and the operation CS by (2.14). The cases where a simple development function $g$ or an accumulating $G$ is contained in the vector are discerned using symbols $V$ or $W$ respectively. (Cf. also equation (7) of Appendix 1.)

\section{RUN-OFF ERROR, BREAK-UP}

\subsection{Problem}

We examined above the impact of the estimation error of the claims reserve $C(t)$ on the profit or loss of the current fiscal year. Now we are asking what is the total error contained in $\boldsymbol{C}(t)$. The problem setting is equivalent to an assumption 
that the insurer would discontinue his business and the claims, which are related to events in year $t$ or earlier, are paid from $C(t)$. As before we do not deal with the estimation problem, instead we assume the reserve rules given in form (1.23).

The sought after error, denoted by $\boldsymbol{R}(t)$ :

$$
\boldsymbol{R}(t)=\boldsymbol{C}(t)-\sum_{s=0}^{T-1} \boldsymbol{X}_{v}(t-s ; s+1, T)
$$

is what is left ( + or - ) when all the claims are finally settled. The yield of interest is taken into account discounting the claims payments to the initial time point $t$.

\subsection{A Solution}

A solution can be found by using a similar technique to that used in Chapter 2 . We give the final formulae:

$$
\begin{aligned}
\operatorname{Var} \boldsymbol{R}(t)= & v_{0} \cdot\left(W_{c I}^{\prime} W_{c m}+V_{I}^{\prime} W_{v m}\right)+v_{q} \cdot\left(W_{c x}^{\prime} W_{c x}+W_{v x}^{\prime} W_{v x}-2 W_{c x}^{\prime} W_{v x}\right) \\
& +\operatorname{CS}\left[W_{c x}, W_{c x}\right]+\operatorname{CS}\left[W_{v x}, W_{v x}\right]-2 \operatorname{CS}\left[W_{c x}, W_{v x}\right]
\end{aligned}
$$

where

$$
\begin{aligned}
W_{c I} & =\left(c_{x}(s) \cdot I_{x}(t-s)\right)^{\prime} \\
W_{c m} & =\left(c_{x}(s) \cdot I_{m}(t-s) G_{m x}(s)\right)^{\prime} \\
W_{v m} & =\left(I_{m}(t-s) \cdot \bar{G}_{m x}(s)\right)^{\prime} \\
W_{c x} & =\left(c_{x}(s) \cdot I_{x}(t-s) \cdot G_{x}(s)\right)^{\prime} \\
W_{v x} & =\left(I_{x}(t-s) \cdot \bar{G}_{x}(s)\right)^{\prime} \\
V_{I} & =\left(I_{x}(t-s)\right)^{\prime}
\end{aligned}
$$

and

$$
\bar{G}_{m x}(s)=\sum_{u=s+1}^{T} v^{2\left(u-s-\frac{1}{2}\right)} \cdot g_{m}(u) \cdot g_{x}(u) .
$$

The index $s$ is now running from 0 to $T-1$. (Cf. also equation (10) of Appendix 1.)

\section{EXAMPLES}

We are going to give some examples of the application of the formulae presented in the previous chapters. Therefore, the data and functions involved must first be specified.

\subsection{The Structure Function}

The structure function $q(t)$ is given by a first order autoregressive time series:

$$
\mathbf{q}(t)-1=a[q(t)-1]+\mathbf{\varepsilon}(t)
$$


where $a$ is a coefficient $0 \leqslant a<1$ and $\varepsilon$, "the noise" is a normally $\left(0, \sigma_{\varepsilon}\right)$ distributed stochastic term. According to the standard formulae of the time series theory (asymptotically)

$$
\begin{aligned}
\sigma_{q} & =\sigma_{\varepsilon} / \sqrt{ }\left(1-a^{2}\right), \\
\gamma(i) & =a^{1} \cdot \sigma_{q}^{2} \quad(i=0,1,2, \ldots) .
\end{aligned}
$$

\subsection{Reserve Rules}

Reserve rules are given according to the following four alternatives:

Premium based rule (abbreviation "pr-bas" in the tables) is defined by

$$
\begin{aligned}
& c_{x}(s)=0 \\
& c_{p}(s)=X_{0} \bar{G}_{x}(s) / P_{0}
\end{aligned}
$$

which is derived by applying the conditions of (1.24).

The "learning" rule (abbreviation "learn") proposed originally by BENKTANDER (1976) and recently developed for reinsurance practice by among others HovineN and the Kansa-companies $(1981,1984)$, is obtained by composing two reserve rules. Firstly, the "premium based" reserve rule is the same as (4.3). The other rule is "claims based", and it is obtained putting $c_{p}$ 's $=0$ in (1.23). Then according to $(1.24) c_{x}(s) \cdot E \boldsymbol{X}(t-s ; 0, s)=E \boldsymbol{X}_{v}(t-s ; s+1, T)$, which implies in the terms of our development functions $c_{x}(s)=\bar{G}_{x}(s) / G_{x}(s)$. The former rule utilizes the past experience contained in the premium $P$ and the latter one the fresh experience received from the accumulating $\boldsymbol{X}(t-s ; 0, s)$. The idea is to combine them first giving more weight to the premium-based rule and later more weight to the claims-based rule. Following an intuitive experience rating principle the reserve for outstanding claims is eventually obtained from them by using the weights

$$
\bar{G}_{x}(s) /\left[\bar{G}_{x}(s)+G_{x}(s)\right] \text { and } G_{x}(s) /\left[\bar{G}_{x}(s)+G_{x}(s)\right] .
$$

Then the following coefficients are the results

$$
\begin{aligned}
& c_{p}(s)=\frac{X_{0}}{P_{0}} \bar{G}_{x}(s)^{2} /\left[\bar{G}_{x}(s)+G_{x}(s)\right] \\
& c_{x}(s)=\bar{G}_{x}(s) /\left[\bar{G}_{x}(s)+G_{x}(s)\right] .
\end{aligned}
$$

The rule is nicknamed "learning" because all the time, while the cohort is aging, it is adapting according to the freshest claims experience.

Optimal rule 1 (opt1) is based on linear regression theory. It is derived seeking coefficients $c_{x}$ which minimise the variance of the (unbiased) break-up error $\boldsymbol{R}(\boldsymbol{t})$ (see (3.1)). Recapitulating the formulae given in Appendix 1 in matrix form we have

$$
c_{x}=M\left(X_{k}\right)^{-1} M\left(X_{k}, X_{u}\right) \mathbb{1}
$$


where, in the terms used above,

$$
\begin{aligned}
M\left(\boldsymbol{X}_{k}\right) & =(\operatorname{Cov}(\boldsymbol{X}(t-s ; 0, s), \boldsymbol{X}(t-u ; 0, u))) \\
M\left(\boldsymbol{X}_{k}, \boldsymbol{X}_{u}\right) & =\left(\operatorname{Cov}\left(\boldsymbol{X}(t-s ; 0, s), \boldsymbol{X}_{v}(t-u ; u+1, T)\right)\right) \\
\mathbb{1} & =(1,1, \ldots, 1)^{\prime}
\end{aligned}
$$

and $s$ and $u$ run from 0 to $T-1$.

Note that only the coefficients $c_{x}$ are defined, not the coefficients $c_{p}$. This is due to the fact that only the former are affiliated with the stochastic terms in $\boldsymbol{X}_{p}$ (see (2.2)) and in $\boldsymbol{R}(t)$ (see (3.1)). The coefficients $c_{p}$ should be determined, if needed, e.g., by the equations (1.24).

Optimal 2 (opt2) is also presented in the Appendix 1, equation (13), based on the optimization of the cohort reserves separately each in turn. Formally, it results from (4.5) if $\gamma(i)$ 's are set equal to zero for $i>0$.

\subsection{The Growth Rates and Indexes}

The growth rates and indexes to be applied in this chapter are assumed to be constants according to the following formulae:

$$
I_{n}(t-s)=r_{n}^{t-s} ; \quad I_{m}(t-s)=r_{m}^{t-s} ; \quad g_{m}(u)=r_{c}^{u}
$$

where $r_{n}$ is the growth factor on the volume parameter $n$, and $r_{m}$ and $r_{c}$ for the inflation between and inside the cohorts, respectively. They as well as the constant rate of interest $(i=i$, above) are given under the pertinent headings in the table.

\subsection{The Run-Off Tails}

The run-off tails are given as three alternatives, the values of $g_{n}(u)$ being according to the ascending $u=0,1, \ldots, T$ as follows:

(a) Long tail $T=12: 0.15,0.25,0.15,0.15,0.10,0.05,0.05,0.02,0.02,0.02$, $0.02,0.01,0.01$ (see Rantala (1984)).

(b) Medium tail $T=3: 0.6,0.2,0.15,0.05$.

(c) Short tail $T=2: 0.8,0.15,0.05$.

The long tail may be suitable for e.g., marine and reinsurance lines, the other two for various classes of domestic business.

\subsection{The Claim Size Distribution}

The scale of the monetary quantities was chosen so that the unity may suitably correspond to about $£ 100000$. We do not need the specification of the claim size d.f., only its lowest three moments, which were assigned the values $0.006,0.001$ and 0.0001 respectively. This means that the average claim size is $£ 6000$. This closely corresponds to the industrial fire distribution that is presented in BPP, $p$. 86 , as a standard example of a rather heterogeneous portfolio provided that the maximum net retention per claim is about $£ 400000$. 


\subsection{Explanation for Table 4.1}

Table 4.1. sets out a number of examples grouped in subsets, each of which is aimed at describing some particular feature of the run-off effects. In order to show the "canals" of the influence the relative magnitudes of the terms in the variance expressions (2.16) and (3.2) are given. The main indicators are the increment $\Delta \sigma_{X}$ of the standard deviation $\sigma_{X}$ compared with the value $\sigma_{0}$ it would have, if no run-off delay were in existence. Furthermore, $\sigma_{X}$ is given as a percentage of the premium income $P_{0}$ (see (1.27)). Similarly the "break-up" $\sigma_{R}$ is presented as a percentage of the expected reserve $C=E C(t)$ (see (1.25)) and of the premium income $P_{0}$.

The effect of the reserve rule is experimented with for cases having varying tail lengths and the size of the collective, measured by $n$. As seen in the decomposition lines $V X i / V X$ and $V R i / V R(i=1,2, \ldots, 8)$ referring to the terms of the formulae (2.16) and (3.2), the Poisson terms (predominantly $V X 1 / V X$ and $V R 2 / V R$ ) determine the outcomes for small collectives, and the structure terms ( $V X 3 / V X$ and $V R i / V R$ for $i=3,4$ ) the behaviour of large collectives as seen, in particular, in the example 11 . The covariance terms $(6,7,8)$ have significance only in large collectives. Note also the existence of negative terms.

Furthermore, the influence of the structure variation is demonstrated providing a possibility to compare the cases where it is present with the cases from which it has been removed (examples 16, 17 and 18). The effect of the autocorrelation was removed from the example 15 , it is to be compared with the case 12 .

\subsection{Some Observations}

Expectedly, the reserve rule has a considerable influence on the run-off variation in the going-concern consideration. The premium based rule reduces the variance in most cases. In fact, it equalizes the fluctuation tops between the consecutive years, it "hides" them in the increased errors in the claims reserve (as evaluated on the break-up bases). The other rules mostly increase the variance from the level which it would have, if there were no run-off delay. This is due to the construction of the reserve, which is affected more immediately by the variation tops in the claims process.

Also the assumptions made on the growth of the portfolio and the inflation rate inside and outside the cohorts have some influence on the behaviour of the system as can be seen when the examples $18 \ldots 22$ are compared with the corresponding other cases in Table 4.1, the growth and inflation data being totally removed from those examples. If, in addition, the structure variation is also removed (example 18), then the run-off delay has no effect in the going-concern variation. It is a clear outcome observing that then both processes $\boldsymbol{X}_{p}(t)$ and $\boldsymbol{X}(t ; 0, T)$ are composed of similar pieces which are each independent compound Poisson processes. Furthermore, cases 16 and 17 are identical, since premium based reserving rules is in fact the "optimal" one, if the structure variation is missing. 







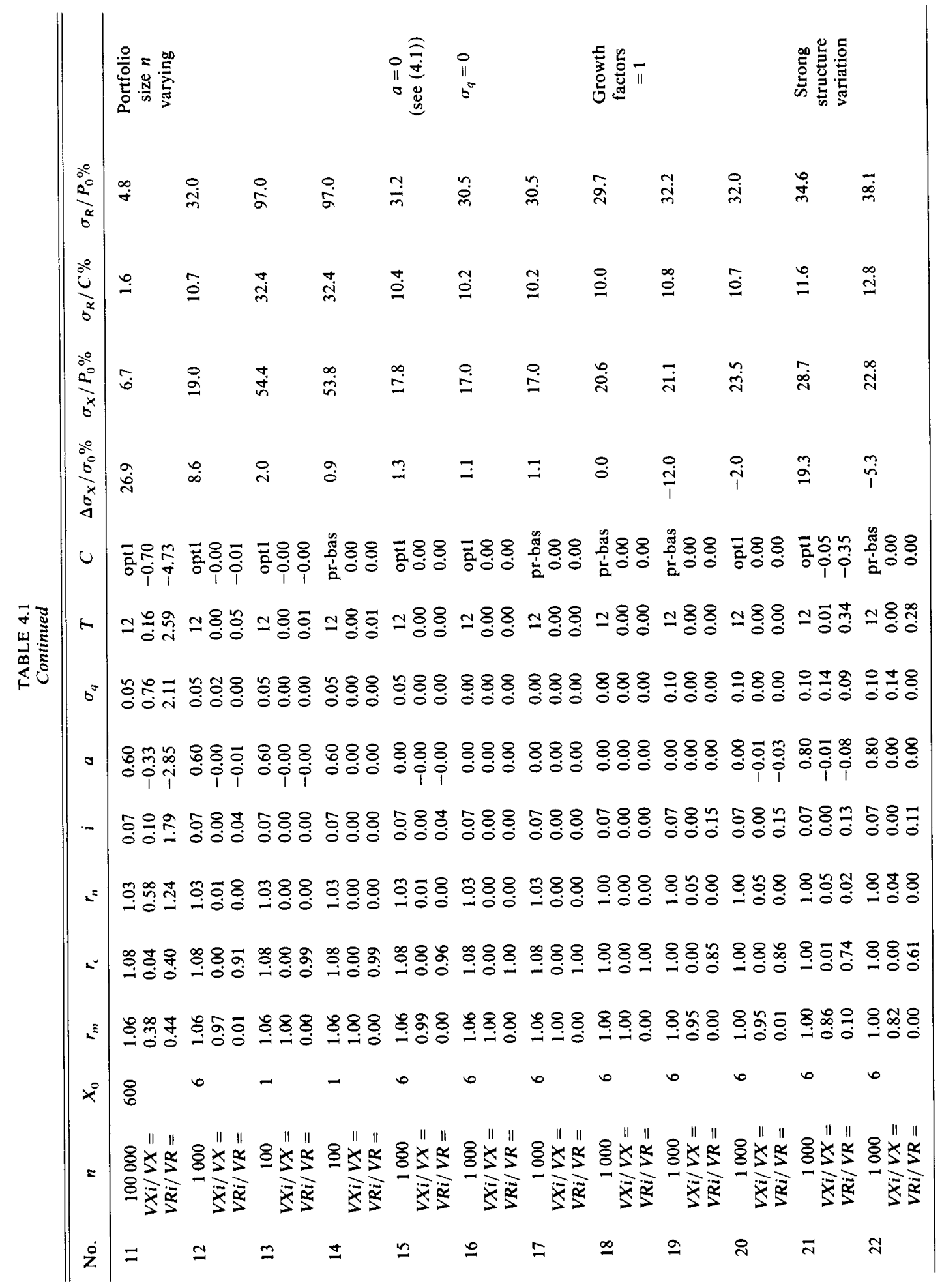


The break-up errors, measured by $\sigma_{R}$, are rather large in small collectives, but somewhat unexpectedly the reserve rules have only a slight influence on it. This is obviously due to the fact that the cohort outcomes equalize each other irrespective of what a rule may be applied.

\subsection{Remarks}

Let us recall anew the restrictions already stated in the introduction. The considerations are subject to specified conditions, excluding among other things the uncertainty involved in the parameter estimation. Therefore, our results should not be understood as describing the total actual fluctuation but only specific components of it. Furthermore, in order to keep this paper in reasonable limits, the autocovariances of the $X_{p}(t)$ or the $R(t)$ variables ( $t$ varying) were not dealt with, nor the correlations between these variable sets. This would be done by using the same building blocks as introduced above. The autocovariance structure of $\boldsymbol{X}_{p}(t)$ may be clearly different from that of the "undelayed" conventional $\boldsymbol{X}(t)$. For instance, if the latter were the compound Poisson process having zero autocovariances, the first $T$ autocovariances of the $X_{p}(t)$ process would be non-zeros, on the contrary to one of the standard assumptions of the conventional risk theory.

\section{SIMULATION}

\subsection{The Problem}

It is also useful to handle the run-off patterns by using the simulation technique. Its merit is in the easy possibility to relax some of the restrictive assumptions made above, e.g., a stochastic inflation and interest can be incorporated into the model without any noticeable complications. We shall draft the method both for the break-up case and for the going-concern consideration, but first a number of definitions and modifications are necessary.

\subsection{Inflation}

Inflation is simulated by the following first order autoregressive time series:

$$
i_{m}(t)-i_{m 0}=a_{m} \cdot\left[i_{m}(t-1)-i_{m 0}\right]+\sigma_{m} \varepsilon_{m}(t)
$$

where

$$
i_{m}(t)=I_{m}(t) / I_{m}(t-1)-1
$$

is the rate of inflation and $I_{m}$ the index of inflation between the cohorts (cf. (1.5)). The variable $\varepsilon$ is a normally $(0,1)$ distributed random number and coefficient $\sigma_{m}$ controls its magnitude.

The equation (5.1) is, with minor modifications, the same as that proposed by Professor Wilkie (1984). The investigation of the inflation index series of numerous countries (Appendix 2) revealed that seem to be lengthy "peaceful" periods 
during which the rates are moderate and obviously distributed in a way which can be appropriately simulated by the original Wilkie formula. But often a peaceful period is broken by a considerably excessive inflation lasting some few years. Because such an event can occasion considerable trouble for the insurance industry, we considered it justified to supplement the model by providing the possibility of giving an additional impact to the "normal" flow of inflation as a "shock". We applied a deterministic shock in some of our examples given in the sequel. However, it is no problem to also make this term stochastic. The shock can be given as an addition to the values which are first generated by equation (5.1) for the whole time horizon or they can be added every year to the current value $i_{m}(t)$ in (5.1) so that they autoregressively affect the subsequent values. The latter procedure creates a tendency for the flow curves to keep soaring for lengthy time periods, which may not be always in good consistency with experience (see Appendix 2). Therefore, we applied the former alternative in our examples.

Another observation was that the rate of inflation is, as a rule, positive and that there seems to be some positive lower limit under which it never or very seldom falls. Therefore, it may be suitable to provide the model with condition:

$$
i_{m}(t) \geqslant i_{\min } \text {. }
$$

Note that the Wilkie model seems to also produce negative rates when his standard data are used.

We used the following standard values for the parameters involved $i_{m 0}=0.06$; $a_{m}=0.7 ; \sigma_{m}=0.015 ; i_{\mathrm{min}}=0.03$.

\subsection{The Interest Rate}

The rate of interest (including also asset appreciations and depreciations) seems to be one of the most problematic links in model building. Its behaviour essentially differs in different countries depending on the size and the character of the capital market in the particular country, on the investment practices of the insurers and on the valuation principles of assets (especially whether or not it is allowed to create buffers by undervaluating them). Professor Wilkie has proposed models for some investment categories based on the British experience. His idea is to make the rates and values dependent on inflation. A high rate of inflation is gradually increasing the rate of the return on investments so that the investors can have inflation losses compensated for in the long run.

We have had to postpone further investigations of the asset models to a future date and have made use of a simplified equation as follows:

$$
i_{i}(t)-i_{t 0}=a_{1} \cdot\left[i_{1}(t-1)-i_{t 0}\right]+\frac{1}{2}\left[i_{m}\left(t-t_{d}\right)+i_{m}\left(t-t_{d}-1\right)-2 i_{m 0}\right]+\sigma_{i} \varepsilon_{1}
$$

where $i_{i}(t)$ is the rate of interest earned on an average for to the whole investment portfolio; $i_{10}$ is the average value of $i_{1}$ (a parameter to be given); $a_{1}$ a coefficient to introduce an autoregressive element into the process; and $\varepsilon_{\boldsymbol{l}}$ is again a normally $(0,1)$ distributed random variable introducing additional noise to the process. 
The second term compensates for the inflation in two years with a time delay $t_{d}$.

We used the following standard values for the parameters $i_{i 0}=0.07 ; a_{i}=0.1$; $\sigma_{i}=0.01$.

Note: Owing to the choice $i_{i 0}>i_{m}$ an expectation of a positive yield of interest in real terms is assumed.

It is convenient to introduce function

$$
I_{i}(t)=\prod_{u=t_{0}}^{t}\left(1+i_{i}(u)\right)
$$

in analogy with $I_{n}(t)(\mathrm{cf} .(5.2)$.

\subsection{Examples}

Figure 5.1 provides examples of the simulation results obtained by means of the above formulae.
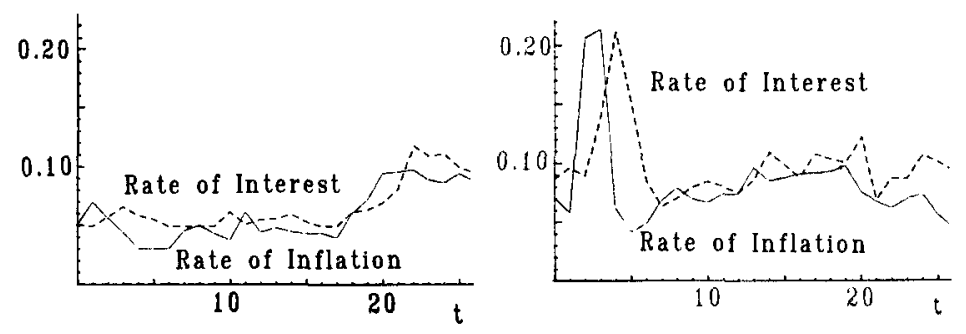

FIGURE 5.1. The rate of inflation and the rate of interest (dotted line) simulated by the algorithms (5.1) and (5.4). A shock of 0.14 was assumed in the right hand side diagram for the years $t=2,3$.

\subsection{Simulation of the Break-up Error}

As the primary building blocks of the simulation procedure the claims amounts $\boldsymbol{X}(t-s ; u, u)$ are needed for various $s$ and $u$ values and they are generated as described in the next items. The claims expenditure, which fall due for payment in the years $t+k(k=1,2, \ldots, T)$ are obtained by summing up the relevant pieces as demonstrated in fig. 5.2. by a shaded pillar. In the simulation of the process in a break-up situation these amounts are paid from the claims reserve $C$ until all of them are settled, the latest ones according to our assumptions in year $t+T$. What is left (+ or - ) of the initial reserve $C(t)$, is just the simulated run-off error. This is obtained by running the algorithm from $k=1$ up to $k=T$ :

$$
C^{\prime}(t+k)=C^{\prime}(t+k-1)-\sum_{s=0}^{T-k} \boldsymbol{V}(t, k) X(t-s ; s+k, s+k)
$$

where $C^{\prime}(t)=C(t)$ and a discounting auxiliary function was introduced in the terms of the interest product $(5.5)$ as follows

$$
\boldsymbol{V}(t, k)=\boldsymbol{I}_{i}(t) / \boldsymbol{I}_{i}\left(t+k-\frac{1}{2}\right) \approx v^{(k-1 / 2)}
$$




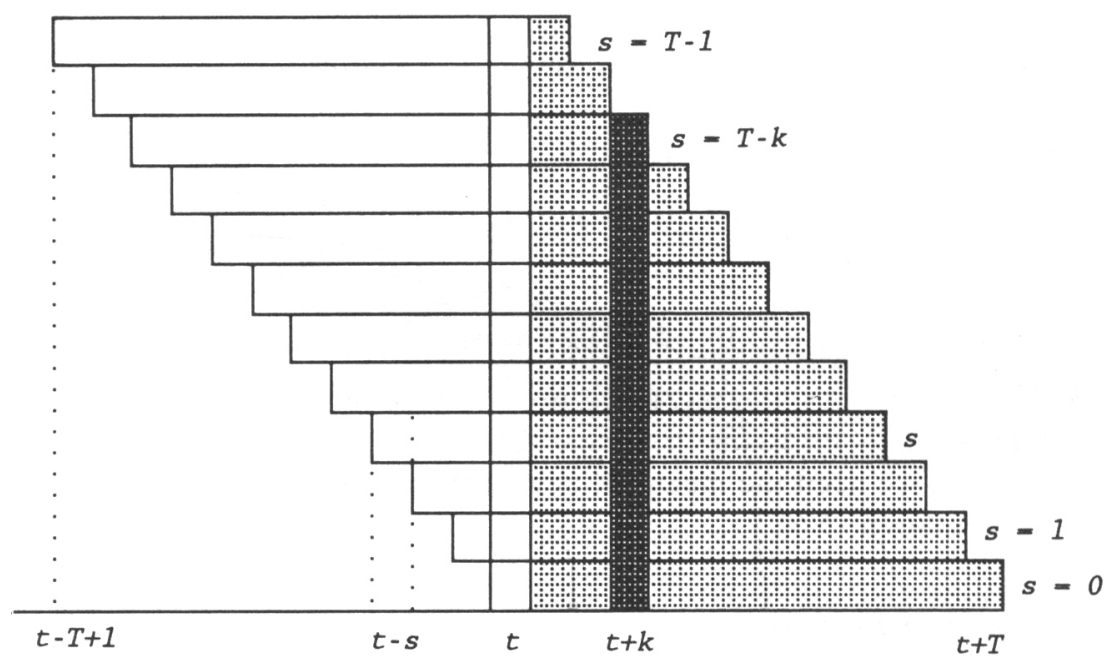

FIGURE 5.2. The run-off pattern composed of the cohorts $t, t-1, \ldots, t-T+1$.

where the last term represents the special case, where the rate of interest is deterministic and constant $v=1 /\left(1+i_{\imath}\right)$. The value to be assigned to the index $I_{i}(\cdot)$ for a half year argument can suitably be taken as the mean of values at the beginning and at the end of the year.

To make the algorithm workable a generator is needed to get the $X$ 's for the summation.

\subsection{A Random Number Generator}

A random number generator for the production of the claims amount may conveniently be of the type, which is described in BPP, Section 6.8.3. It is based on the assumption that the distribution function $F(X)$ of the total amount of claims inherent from some specified collective and period can be approximated by a formula of the type

$$
F(X)=N(f(X))
$$

where $N$ is the standard normal d.f. and $f$ is some suitable transformation which replaces the variable by (as far as possible) a symmetric one (see BPP, Section 3.11) The well-known NP-approximation is an example belonging to this family of approximations. In the sequel we use the Wilson-Hilferty approximation (BPP, item $3.5 b$ ). Then compound Poisson distributed random numbers can be generated as follows:

(1) Generate a normally $(0,1)$ distributed number (BPP, item 6.8f) $r_{n}$.

(2) Do the transformation $r_{v}=f^{-1}\left(r_{n}\right)$ so arriving at a random number, which has the mean, st. deviation and skewness 0,1 and $\gamma_{X}$ respectively. 
(3) $r_{X}=m_{X}+r_{X} \sigma_{X}$ is then the requested (approximately) compound Poisson distributed random number having the mean, st. deviation and skewness $m_{X}, \sigma_{X}$, $\gamma_{X}$.

The Wilson-Hilferty transformation is

$$
r_{v}=f^{-1}\left(r_{n}\right)=c_{1} \cdot\left(r_{n}-c_{2}\right)^{3}-c_{3}
$$

where, denoting $g=\frac{1}{6} \gamma_{X}$.

$$
c_{1}=g^{2} / 3 ; \quad c_{2}=g-1 / g ; \quad c_{3}=2 / g .
$$

(See BPP, equation (3.5.14), p. 71, a report on this and related approximations will be published by Pentikäinen in near future)).

Furthermore, we need the mean, st. deviation and skewness for each relevant $\boldsymbol{X}$ in the summation (5.6).

\subsection{The Basic Characteristics}

We recapitulate the formulae of the above mentioned basic characteristics:

$$
\begin{aligned}
& m_{X}(t-s ; u, u)=n(t-s ; u, u,) m(t-s ; u, u) \\
& \sigma_{X}(t-s ; u, u)=\sqrt{ }\left[n(t-s ; u, u) \cdot a_{2}(t-s ; u, u)\right] \\
& \gamma_{X}^{\prime}(t-s ; u, u)=\mu_{3}(t-s ; u, u) / \sigma_{X}(t-s ; u, u)^{3}
\end{aligned}
$$

(see BPP, equation (3.3.9), p. 54).

The variable $n(t-s ; u, u)$ can be obtained from (1.3) without any need for modifications. The structure variable $q(t-s)$ is to be generated separately for each cohort (and for each realization of the simulation process). For instance, the autoregressive algorithm (4.1) or any similar one can be used. The noise term $\varepsilon(t)$ may be generated assuming it to be normally distributed.

The development function $g_{m}(u)$ will be modified separating the influence of inflation and the possible change in the real value of the delayed claims, the latter given by a function $g_{0}(u)$. In our simulations we employ the formula

$$
\boldsymbol{g}_{m}(u)=g_{0}(u) \boldsymbol{I}_{m}(t-s+u) / \boldsymbol{I}_{m}(t-s) \text {. }
$$

Also $g_{0}(u)$ can be made cohort dependent or dependent on the current time $t+k$, if there is found a justification for it.

The characteristics needed in the generator can now be written as follows

$$
\begin{gathered}
\boldsymbol{m}_{X}(t-s ; u, u)=m \cdot \boldsymbol{I}_{m}(t-s) \cdot g_{0}(u) \cdot \boldsymbol{I}_{m}(t-s+u) / \boldsymbol{I}_{m}(t-s) \\
=m \cdot \boldsymbol{I}_{m}(t-s+u) \cdot g_{0}(u) \\
\boldsymbol{\sigma}_{X}(t-s ; u, u)=\boldsymbol{I}_{m}(t-s+u) \cdot g_{0}(u) \cdot \sqrt{ }\left[n(t-s ; u, u) \cdot a_{2}\right] \\
\boldsymbol{\gamma}_{X}(t-s ; u, u)=\frac{a_{3}}{a_{2}^{3 / 2}} \cdot \frac{1}{\sqrt{ }(\boldsymbol{n}(t-s ; u, u))}
\end{gathered}
$$

The monetary quantities are obtained at the level of year $t-s+u$. 


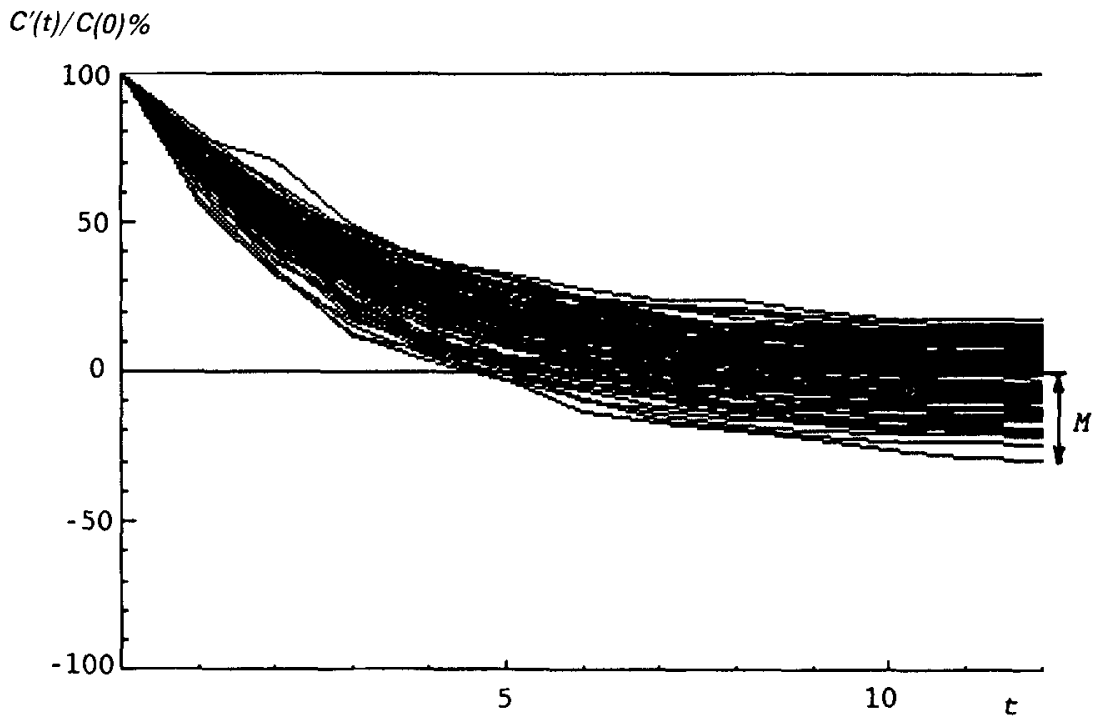

Figure 53 A simulated run-off pattern The data are the same as in the case 12 in Table 41 , but inflation is now made stochastic Sample size 100 Mean $=05 \%$ (the theoretical value $=0$ in Table

41 ) and standard deviation $=106 \%(107 \%$ in Table 41$)$ The "safety margın" $M \approx 30 \%$

\section{$C^{\prime}(t) / C(0) \%$}

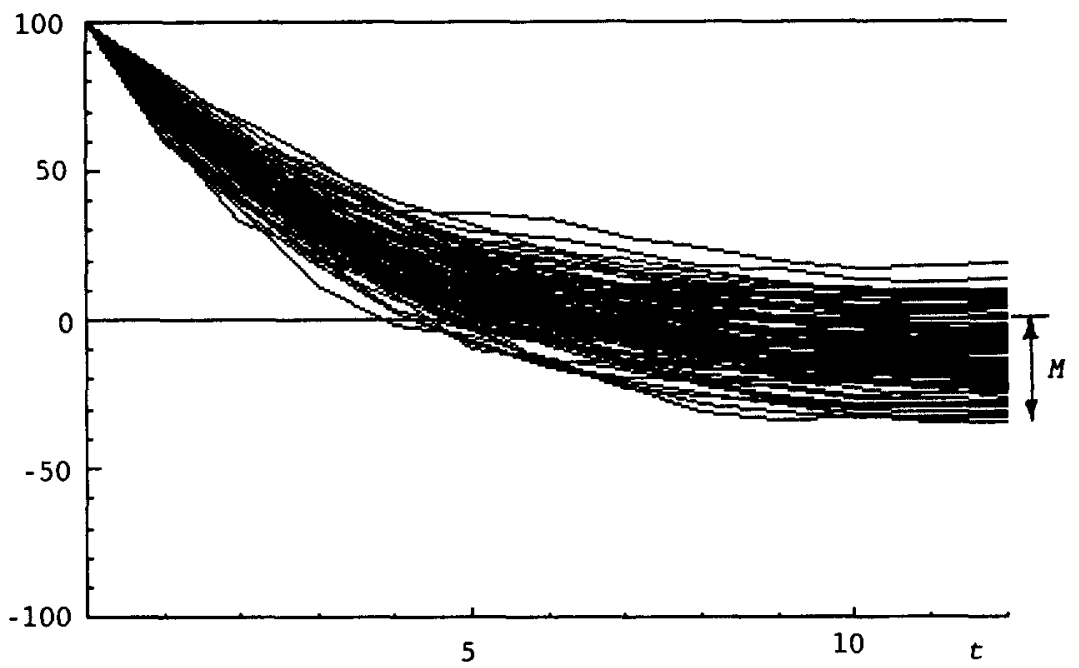

Figure 54 The same run-off pattern as in Fig 53 but now assuming an inflation shock of $14 \%$ in addition to the simulated normal inflation in years $t+3$ and $t+4$ Mean $-22 \%$, standard deviation $119 \%$ and the margin $M \approx 37 \%$ 


\subsection{Examples}

Figures 5.3 and 5.4 display two examples of the simulation outcomes of break-up processes.

In order to have the results comparable with the deterministic value of Table 4.1 the interest was kept deterministic, the stochasticity as introduced in item 5.3 being incorporated in the going-concern simulation in the next following items.

That part $M$ of the variation range of the residue which falls below the zero line in figs. 5.1 and 5.2 can be used as an estimate for the margin necessary to be incorporated into the claims reserve, if it is required that the run-off' of the reserve in a break-up situation may not result in a negative residue by a desired probability.

\subsection{The Simulation of the Going-Concern Process}

In order to arrive at a conception of the influence of the run-off impact the ratio

$$
\boldsymbol{x}(t)=\boldsymbol{X}_{p}(t) / \boldsymbol{B}(t)
$$

will be simulated for some time period. Here $\boldsymbol{X}_{p}$ is the claims expenditure as defined by (2.1) and

$$
B(t)=P(t)+i_{1}(t) \cdot \frac{1}{2}\left[C^{\prime}(t)+C^{\prime}(t-1)\right]
$$

is, what can be called underwriting income, the premium income (1.26) supplemented by the interest earned by the claims reserve (5.1).

In order to simulate, so far as possible, the conventional income statements it is assumed that the incomes are received and the payments made in the middle of each year. Therefore, the premium income differs slightly from that defined in item 1.15. The latter should now be multiplied by $v^{1 / 2}$ to make the process consistent.

Another indicator is the relative run-off error

$$
\boldsymbol{r}(t)=\boldsymbol{R}(t) / \boldsymbol{B}(t)
$$

where $\boldsymbol{R}$ is the error as defined by (3.1).

Both $\boldsymbol{x}$ and $\boldsymbol{r}$ are composed of a number of claims amounts $\boldsymbol{X}(t-s, u, u)$ as it can be found in similar way as described above for the break-up case.

\subsection{Graphic Examples}

Figure 5.5 exhibits simulated curves of the above target ratios $\boldsymbol{x}$ and $\boldsymbol{r}$. The former was simulated in parallel omitting the run-off effect $\left(\boldsymbol{x}_{0}\right)$ and taking it into account $(\boldsymbol{x})$. The former is, in fact, what the claims amount would be if it were possible to evaluate the outstanding claims without any estimation error. The corresponding $r$ curve is set out. We see how some part of the actual fluctuation of the annual claims amount $\boldsymbol{x}$ is smoothed away from $x_{0}$ and "hidden" in the fluctuating run-off error $r$. This feature is characteristic of the premium based reserve rule (cf. item 4.3) which was applied in this example. 


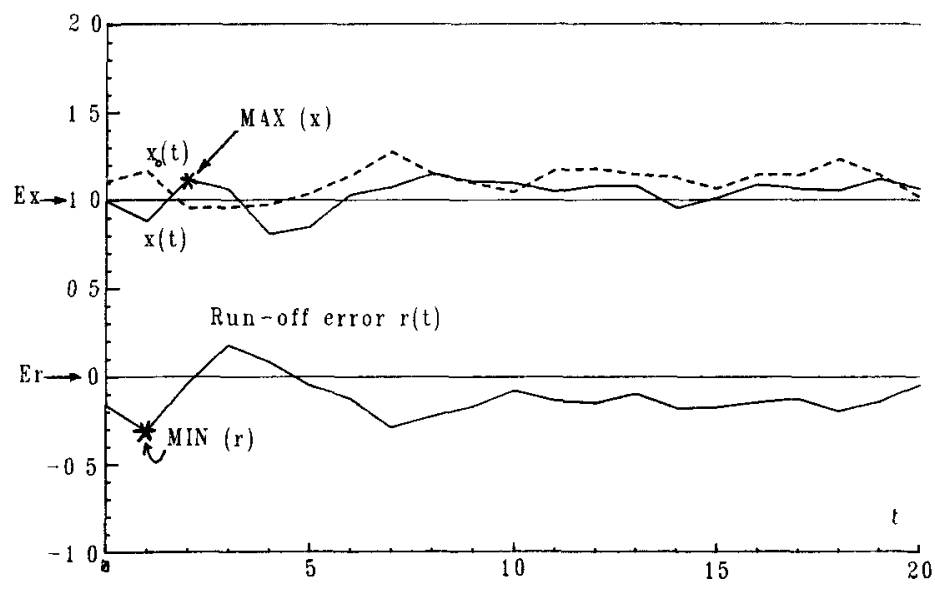

FIGURE 5.5. A simulated fow of the claims ratios assuming that the run-off error is $=0\left(x_{0}\right)$ and taking it into account $(x)$ according to our standard assumption. The corresponding relative run-off error $\boldsymbol{r}$ is set out for the same process. A shock as described in item 5.2 was assumed. The asterisks indicate the maximum or minimum point of the curve concerned and they will be adapted as indicators of the volatility of the process as will be later explained.

Note that according to the definition of the run-off error (3.1) a negative value of it indicates a case where the reserve of outstanding claims was not sufficient to meet the simulated claims.

A survey of the process concerned can be obtained repeating the simulation numerous times according to the Monte Carlo method as we already did in the case of the break-up process. Figure 5.6 displays an example of a process with and without an inflation shock.

\subsection{Tabular Results}

If the effect of different reserve rules and other relevant issues are to be evaluated and compared, the differences in the results often are difficult to ascertain from diagrams such as exemplified above. Therefore, it is useful to present the simulation results also in a numerical form. We experimented with the following idea. First the maximum value of $\boldsymbol{x}(t)$ and the minimum of $\boldsymbol{r}(t)(t$ running over the test period) are picked up from the curves of Fig. 5.5. They are earmarked by asterisks.

The Monte Carlo process as that of Fig. 5.6. then produces a sample of the distribution of these quantities MAX $[x(t)]$ and MIN $[\boldsymbol{r}(t)]$ and an approximate evaluation for the risk probabilities

$$
\operatorname{Prob}\left\{\operatorname{MAX}[x(t)]>x_{e}\right\}=e_{x} \text { and } \operatorname{Prob}\left\{\operatorname{MIN}[r(t)]<r_{e}\right\}=e_{r} .
$$

If information about the range of adverse fluctuation is needed in a concise form, the limits $x_{e}$ and $r_{e}$ may be useful indicators, when the $e$ 's are fixed at some suitable level, say 0.01 or 0.05 . 


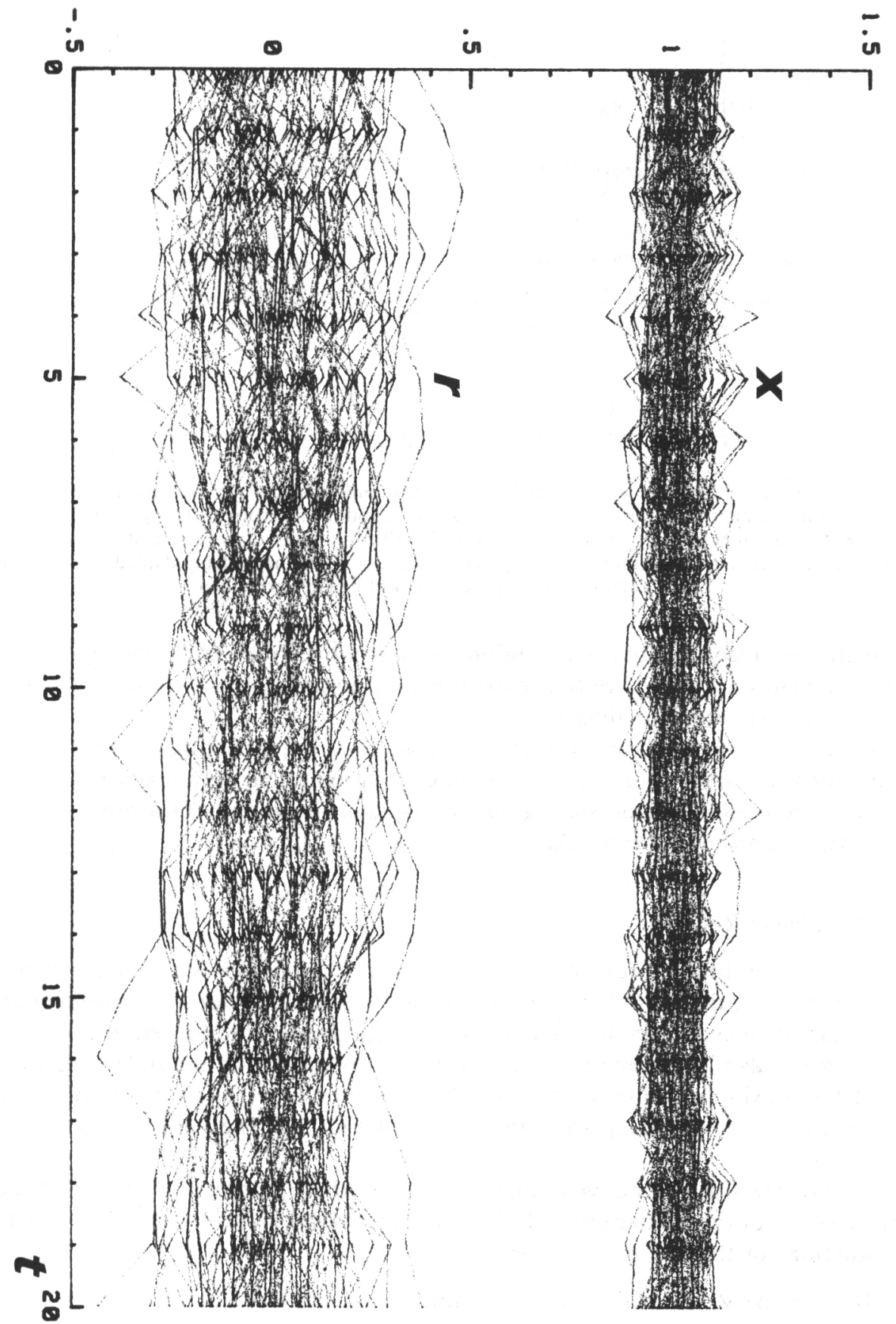

FIGURE 5.6. Simulated going-concern processes. The data are the same as in the case 2 of Table 4.1. An inflation shock such as shown in Fig. 5.1 is assumed in the lower diagram. Note, when comparing the outcomes of the table and the diagrams, that the denominators of $\boldsymbol{x}$ and $\boldsymbol{r}$ are slightly different as seen in the heading of Table 4.1. and in equations (5.13) and (5.15). 




FIGURE. 5.6 (Continued) 
Note that the conventional indicators such as the standard deviation or its multiples may not be suitable in this context, if a temporary inflation shock or business cycles are included in the process.

Table 5.1 exhibits some few examples of the above indicators. The tail is the run-off period as defined in item 1.2. The basic data are as given above. Test period 10 years, sample size 1000 .

TABLE 5.1

Simulation Results, GOING-CONCERN

\begin{tabular}{cccccccccc}
\hline \hline No. & $n$ & Tail & Reserve & Inflation & Interest & $x_{001}$ & $x_{\mathbf{0} 05}$ & $r_{001}$ & $r_{\mathbf{0 5}}$ \\
\hline 1 & 10000 & 0 & - & - & - & 1.27 & 1.23 & - & - \\
2 & 10000 & 12 & learn & det, no shock & det & 1.25 & 1.21 & -0.30 & -0.28 \\
3 & 10000 & 12 & learn & stoch. no shock & stoch & 1.27 & 1.23 & -0.36 & -0.28 \\
4 & 10000 & 12 & learn & stoch + shock & stoch & 1.32 & 1.27 & -0.57 & -0.50 \\
5 & 10000 & 12 & pr-bas & stoch + shock & stoch & 1.25 & 1.21 & -0.60 & -0.50 \\
6 & 10000 & 12 & Opt1 & stoch + shock & stoch & 1.34 & 1.28 & -0.56 & -0.47 \\
7 & 10000 & 3 & learn & stoch + shock & stoch & 1.31 & 1.26 & -0.26 & -0.23 \\
8 & 100 & 12 & learn & stoch +shock & stoch & 1.98 & 1.95 & -0.98 & -0.93 \\
\hline
\end{tabular}

\section{SOME CONCLUDING REMARKS}

6.1. The above examples were intended only to demonstrate the methods described above, the direct calculations and the simulations. To make an analysis and to obtain a view of the behaviour of various run-off patterns was beyond the scope of this paper. However, some very tentative observations can be quoted.

6.2. In the case of going-concern considerations the effect of the run-off errors on the conventionally counted claims expenditures seems to be noticeable only when the run-off tail is rather long and the rate of inflation varies widely. The effect is likely to be greatly strengthened if the rate of interest cannot fairly flexibly follow the movements of the rate of inflation, but these aspects were not studied.

The size of the portfolio seems to be one of the relevant factors as is seen comparing example rows 4 and 8 in Table 5.1 .

6.3. Our plan is to extend the studies to also cover the solvency margin, which is constituted as the accumulated profits or losses. It is obvious that aspects such as are applied in control theories concerning the continuous adjusting of premiums and other pertinent quantities according to the ever present changing state of the process may have an important role (RANTALA 1984). As already noted another planned extension is to incorporate the parameter estimation procedure into the model.

6.4. Expectedly, the run-off phenomenon can also play a significant role in the evaluation of the solvency conditions of insurers. The effect depends essentially on whether the break-up principle or the going-concern alternative should be 
adopted as the basic philosophy, because in the case of a break-up situation the run-off error is to be taken into account in one way or another in full whereas in the going-concern consideration it may be sufficient to only regard its influence on the loss ratio $x$. A discussion of whether or when the break-up basis is appropriate to be accepted falls beyond the scope of this paper (the topic is discussed in the transactions of the Conference on Insurance Solvency, PENTIKÄINEN (1986)).

6.5. The modelling of the rate of interest (asset risk) is one of the points which was left open for future studies. A weakness of our tentative equation (5.4) is that it does not regard the possibility of such a major plunge in the yield, as that which occurred in some countries when the values of equities and some assets dropped immensely in the mid-1970s (not to speak of the Great Depression at the beginning of 1930s). Possibly this shortcoming can be compensated for by inserting into the model a deterministic or stochastic adverse impact similar to the "shock" we incorporated into the inflation equation (5.1).

\section{ACKNOWLEDGEMENT}

Attention to the significant role of the run-off risk and related asset risks in solvency analyses has been focused on by British actuaries in recent years, CouTTS et al. (1984), the Solvency Working Party chaired by DAYKIN (1984), WILKIE (1984), DAYKIN and BERnstein (1985), etc. We have had the opportunity to co-operate in their work, which is related to our own solvency studies (1982). This paper was inspired very much by the desire to serve as a contribution in our joint efforts to explore these topics, which obviously have great importance for the future development of the application-oriented risk theory, as well as for the solvency considerations, reserve calculations, and also for rating practices.

\section{REFERENCES}

Beard, R. E., Pentikäinen, T. and Pesonen, E. (1984) Risk Theory. Chapman \& Hall, London. Benktander, G. (1976) An Approach to Credibility in Calculating IBNR for Casualty Excess Reinsurance. The Actuarial Review.

Coutts, S. M., Devitt, E. R. F. and Ross, G. A. F. (1984) A Probabilistic Approach to Assessing the Financial Strength of a General Insurance Company. Transactions of the 22nd International Congress of Actuaries.

DAYKIN, C. (1984) The Development of Concepts of Adequacy and Solvency in Non-life Insurance in the EEC. Transactions of the 22nd International Congress of Actuaries.

Daykin, C., DevitT, E. R. F., Khan, M. R. and MCCaughan, J. P. (1984) The Solvency of General Insurance Companies. Journal of the Institute Actuaries, 111, II.

DAYKIN, C. and Bernstein, G. (1985) A Simulation Model to Examine Questions of Solvency in the Light of Asset and Run-off Risks. ASTIN Colloquium.

GISG Working PARTy (1983) Report on Outstanding Claims Reserve. Discussion paper.

Hovinen, E: (1981) Additive and Continuous IBNR. Presented at ASTIN Colloquium.

Hovinen, E. (1984) Technical Reserves in KANSA Group. Report printed by Kansa Corporation, Helsinki.

Karlsson, J. C. (1974). A Stochastic Model for Time-lag in Reporting Claims. Journal of Applied Probability, 11, 2. 
Nationale Nederlande (1981) Loss Reserving Methods. Actuarial Studies 1.

NorberG, R. (1985) A Contribution to Modelling of IBNR Claims. Statistical Research Report 4, University of Oslo.

Pentikäinen, T. (1986) On the Solvency of Insurers. Presented at the International Conference on Insurance Solvency, Philadelphia.

PENTIKÄINEN, T. and RANTALA, J. (1982) Solvency of Insurers and Equalization Reserves. Insurance Publishing Company, Helsinki.

PhILIPSON, C. (1965) Evaluation of Outstanding Liabilities. ASTIN Bulletin.

RANTAla, J. (1983) Estimation of IBNR Claims. Acta Universitatis Tamperenses, A, 153.

Rantala, J. (1984) An Application of Stochastic Control Theory to Insurance Business. Acta Universitatis Tamperensis, A, 164 (Academic dissertation).

TAYLOR, G. C. (1986) Claims Reserving in Non-Life Insurance. North-Holland, Amsterdam.

Wilkie, A. D. (1984) Using a Stochastic Model to Estimate the Distribution of Real Rates Return on Ordinary Shares. Transactions of the 22nd Congress of Actuaries.

\section{APPENDIX 1}

MATRIX PRESENTATION FOR $\operatorname{Var}\left(\boldsymbol{X}_{p}(t)\right)$ AND $\operatorname{Var}(\boldsymbol{R}(t))$, OPTIMAL CLAIMS RESERVES

Let

$$
\text { 1. } \operatorname{Var}\left(\boldsymbol{X}_{p}(t)\right)
$$

$$
\begin{gathered}
\quad \boldsymbol{X}_{1}(t)=(\boldsymbol{X}(t ; 0,0), \boldsymbol{X}(t-1 ; 1,1), \ldots, \boldsymbol{X}(t-T ; T, T))^{\prime} \\
\quad \boldsymbol{X}_{2}(t)=(\boldsymbol{X}(t ; 0,-1), \boldsymbol{X}(t-1 ; 0,0), \ldots, \boldsymbol{X}(t-T ; 0, T-1))^{\prime} \\
a=(a(0), a(1), \ldots, a(T))^{\prime} ; \quad b=(b(0), b(1), \ldots, b(T))^{\prime} .
\end{gathered}
$$

(for notations see Chapter 2 ). Then (2.4) can be written briefly as

$$
\boldsymbol{X}_{p}(t)=a^{\prime} \boldsymbol{X}_{1}(t)+b^{\prime} \boldsymbol{X}_{2}(t)+\text { non-stochastic terms. }
$$

Hence

$$
\operatorname{Var}\left(\boldsymbol{X}_{p}\right)=a^{\prime} M\left(\boldsymbol{X}_{1}\right) a+b^{\prime} M\left(X_{2}\right) b+2 a^{\prime} M\left(X_{1}, X_{2}\right) b,
$$

where $M(X, Y)$ denotes the covariance matrix of random vectors $X$ and $Y$ and $M(X)=M(X, X)$ and the arguments are dropped from the notations. The necessary covariances can be obtained from (1.19) and (2.9). In fact, we have

$$
\begin{gathered}
\frac{M\left(X_{1}\right)}{X_{0}^{2}}=v_{p} \cdot D\left(I_{x} I_{m} g_{m x}\right)+D\left(I_{x} g_{x}\right) M(q) D\left(I_{x} g_{x}\right), \\
\frac{M\left(X_{2}\right)}{X_{0}^{2}}=v_{p} \cdot D\left(I_{x} I_{m} G_{m x}\right)+D\left(I_{x} G_{x}\right) M(q) D\left(I_{x} G_{x}\right), \\
\frac{M\left(X_{1}, X_{2}\right)}{X_{0}^{2}}=D\left(I_{x} g_{x}\right) M(q) D\left(I_{x} G_{x}\right),
\end{gathered}
$$

where $v_{p}=v_{0} / X_{0}^{2}$ and $D(Z)$ denotes a diagonal matrix with the elements of the vector $Z$ on the diagonal. The covariance matrix $M(q)$ is

$$
M(\boldsymbol{q})=\left(\begin{array}{cccc}
\sigma_{q}^{2} & \gamma(1) & \ldots & \gamma(T) \\
\gamma(1) & \sigma_{q}^{2} & \ldots & \gamma(T-1) \\
\vdots & & \ldots & \\
\gamma(T) & \gamma(T-1) & \ldots & \sigma_{q}^{2}
\end{array}\right)
$$

By reordering we obtain

$$
\begin{aligned}
\frac{\operatorname{Var}\left(\boldsymbol{X}_{p}\right)}{X_{0}^{2}}= & v_{p}\left[a^{\prime} D\left(I_{x} I_{m} g_{m x}\right) a+b^{\prime} D\left(I_{x} I_{m} G_{m x}\right) b\right] \\
& +a^{\prime} D\left(I_{x} g_{x}\right) M(q) D\left(I_{x} g_{x}\right) a+b^{\prime} D\left(I_{x} G_{x}\right) M(q) D\left(I_{x} G_{x}\right) b \\
& +2 a^{\prime} D\left(I_{x} g_{x}\right) M(q) D\left(I_{x} G_{x}\right) b .
\end{aligned}
$$


Let

2. $\operatorname{Var}(\boldsymbol{R}(t))$

$$
\begin{aligned}
& \boldsymbol{X}_{k}(t)=(\boldsymbol{X}(t ; 0,0), \boldsymbol{X}(t-1 ; 0,1), \ldots, \boldsymbol{X}(t-T+1 ; 0, T-1))^{\prime} \\
& \boldsymbol{X}_{u}(t)=\left(\boldsymbol{X}_{v}(t ; 1, T), \boldsymbol{X}_{v}(t-1 ; 2, T), \ldots, \boldsymbol{X}_{v}(t-T+1 ; T, T)\right)^{\prime}
\end{aligned}
$$

In order to have a linear and unbiased total claims reserve the reserve formula should be of the form

$$
\boldsymbol{C}=c_{x}^{\prime} \boldsymbol{X}_{k}-c_{x}^{\prime} E \boldsymbol{X}_{k}+\nabla^{\prime} E \boldsymbol{X}_{u}
$$

where $1=(1,1, \ldots, 1)^{\prime}$ and $E X_{k}$ and $E X_{u}$ are given by (1.14) and (1.21). Then the variance of the run-off error

$$
\boldsymbol{R}=\boldsymbol{C}-\mathbb{\nabla}^{\prime} \boldsymbol{X}_{u}
$$

can be obtained from

$$
\operatorname{Var}(\boldsymbol{R})=c_{x}^{\prime} M\left(\boldsymbol{X}_{k}\right) c_{x}+\mathbb{v}^{\prime} M\left(\boldsymbol{X}_{u}\right) \mathbb{1}-2 c_{x}^{\prime} M\left(\boldsymbol{X}_{k}, \boldsymbol{X}_{\mathbf{u}}\right) \mathbb{1}
$$

where the covariances needed are given by $(1.19),(2.9)$ and $(2.11)$ with slight modifications to take into account the effect of discounting. The covariance matrices in (10) can be expressed as functions of $v_{p}$ and $M(q)$ in the similar way as in (7).

\section{The optimal claims reserve}

It follows from the theory of linear regression models that the expected mean square error $E\left(\boldsymbol{R}^{2}\right)=$ $\operatorname{Var}(\boldsymbol{R})$ of the claims reserve is minimized when

$$
\left.c_{x}=M\left(X_{k}\right)^{-1} M\left(X_{k}, X_{u}\right)\right\rceil
$$

Note that in our general framework $c_{x}$ depends on current time $t$ due i.a. to time-dependent Poisson-variance.

In some cases it may be desirable to compute an optimal (in the mean square error sense) claims reserve for each cohort separately. Then the coefficients $c_{x}(s)$ are

$$
\begin{aligned}
c_{x}(s) & =\frac{\operatorname{Cov}\left(\boldsymbol{X}(t-s ; 0, s), \boldsymbol{X}_{v}(t-s ; s+1, T)\right)}{\operatorname{Var}(\boldsymbol{X}(t-s ; 0, s))} \\
& =\frac{v_{q} \cdot I_{x}(t-s) G_{x}(s) \bar{G}_{x}(s)}{v_{0} \cdot I_{m}(t-s) G_{m x}(s)+v_{q} \cdot I_{x}(t-s) G_{x}(s)^{2}},
\end{aligned}
$$

which in the general case depends on $t$. However, if $I_{n} \equiv 1$ (which implies $I_{m}=I_{x}$ ) then $c_{x}(s)$ is independent of $t$ and can be written as

$$
c_{x}(s)=\frac{G_{x}(s) \cdot \bar{G}_{x}(s)}{v_{0} / v_{q} \cdot G_{m x}(s)+G_{x}(s)^{2}} .
$$

If further $q_{m}(u) \equiv 1$ then $G_{m x} \equiv G_{x}$ and (13) reduces to

$$
c_{x}(s)=\frac{\bar{G}_{x}(s)}{v_{0} / v_{q}+G_{x}(s)},
$$

which, in fact, corresponds to the traditional linear credibility coefficient of credibility theory.

RANTALA (1984) contains a numerical example on the differences between (11) and (12). 


\section{APPENDIX 2 \\ CONSUMER PRICES}

Percentage changes from previous year
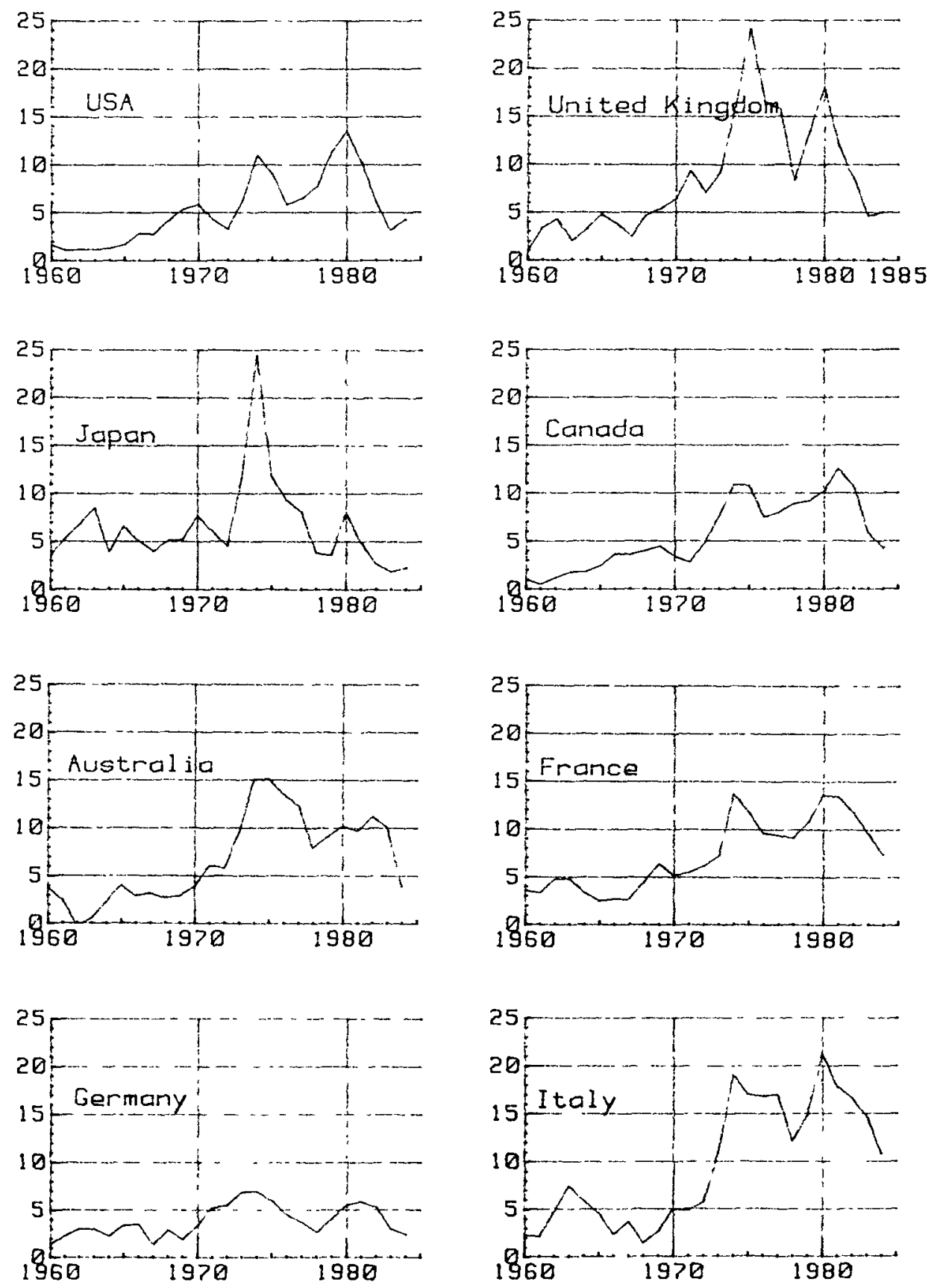

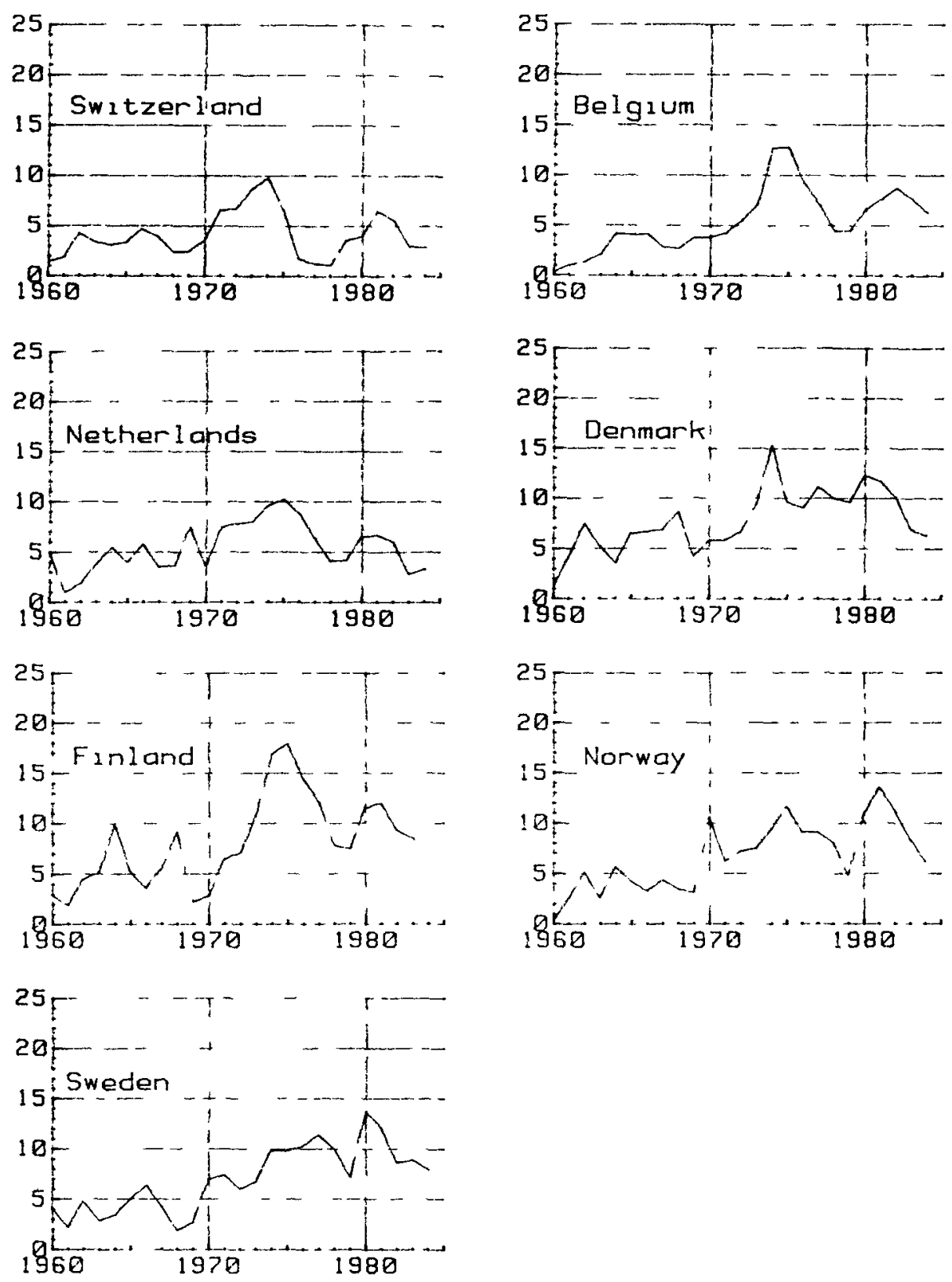

Teivo Pentikainen

Kasavuorentle 12 C 9, 02700 Kauniainen, Finland

JUKKa RANTALA

The Ministry of Soctal Affairs and Health, Bulevard 28,01200 Helsinkı 12, Finland 